\title{
THE INCOMPATIBILITY OPERATOR: FROM RIEMANN'S INTRINSIC VIEW OF GEOMETRY TO A NEW MODEL OF ELASTO-PLASTICITY
}

\author{
SAMUEL AMSTUTZ AND NICOLAS VAN GOETHEM
}

\begin{abstract}
The mathematical modelling in mechanics has a long-standing history as related to geometry, and significant progresses have often been achieved by the invention of new geometrical tools. Also, it happened that the elucidation of practical issues led to the invention of new scientific concepts, and possibly new paradigms, with potential impact far beyond. One such example is Riemann's intrinsic view in geometry, that offered a radically new insight in the Physics of the early 20th century. On the other hand, the rather recent intrinsic approaches in elasticity and elasto-plasticity also share this philosophical standpoint of looking from inside, i.e., from the "manifold" point of view. Of course, this approach requires smoothness, and is thus incomplete for an analyst. Nevertheless, its first aim is to highlight the concepts of metric, curvature and torsion; these notions are addressed in the first part of this survey paper. In a second part, they are given a precise functional meaning and their properties are studied systematically. Further, a novel approach to elasto-plasticity constructed upon a model of incompatible elasticity is designed, carrying this intrinsic spirit. The main mathematical object in this theory is the incompatibility operator, i.e., a linearized version of Riemann's curvature tensor. So far, this route not only has led the authors to a new model with a solid functional foundation and proof of existence results, but also to a framework with a minimal amount of ad-hoc assumptions, and complying with both the basic principles of thermodynamics and invariance principles of Physics. The questions arising from this novel approach are complex and intriguing, but we believe that the model is now sufficiently well posed to be studied simultaneously as a problem of mathematics and of mechanics. Most of the research programme remains to be done, and this survey paper is written to present our model, with a particular care to put this approach into a historical perspective.
\end{abstract}

\section{ON THE ORIGIN OF CURVATURE IN SCIENCE AND THE BIRTH OF INTRINSIC VIEWS}

A first axiomatisation of geometry can be assigned to Euclid of Alexandria (circa 300 B.C.) with The elements gathering the knowledge of the time in planar and solid geometry. A little later, a remarkable calculation of the perimeter of the Earth by Eratosthenes of Cyrene provided a first indirect measure of the curvature of the Earth. In the same third century B.C. Archimedes and Apollonius developed the first ideas of plane curvature in the theory of conic sections. The problem of mapping the earth on a planisphere raised the first projections by the geographers of the Antiquity, the principal one being Ptolemy (150 B.C.). Euler, in his Recherches sur la courbure des surfaces (1767) [23], laid the foundations of the differential geometry of surfaces. Two centuries before, in the age of discoveries arose the problem of navigation with planar representations of the Earth. A precursor in this field is the Portuguese mathematician Pedro Nunes, who distinguished the great circles from the rhumb lines, and in his Opera [56] of 1566 proposed the rectification of the rhumb line in the sea charts. Three years later, the Belgian mathematician and cartographer Gérard Mercator produced the first wall map of the world, that he called New and more complete representation of the terrestrial globe properly adapted for its use in navigation, with the conformal projection (i.e., angle-preserving) that now bears his name. In a certain sense, this can be regarded as the dawn of the co-existence of the intrinsic representation (as the map, with its intrinsic metric used by Mercator, preserves the shapes but not the areas) with the embedding view (the map can

2010 Mathematics Subject Classification. 35J48,35J58,49S05,49K20,74C05,74G99,74A05,74A15, 80A17.

Key words and phrases. Elasticity, plasticity, strain incompatibility, dislocations, virtual work, objectivity. 
be embedded in the Euclidean space, yielding the terrestrial globe). The next crucial achievement arose 250 years later with the Gauss Theorema Egregium [28], stating that the scalar curvature is indeed an intrinsic property of a surface, in the sense that it can be computed only by local measurements of distances independently of the ambient space in which the surface is embedded.

The generalization to higher dimensions of Gauss' work for surfaces was initiated by one of his students, Bernhard Riemann in 1854 [58]. Riemann was the first to introduce the notion of differentiable manifold and of a quadratic form (the so-called Riemannian metric) in order to compute the length of a generalized notion of curve. Moreover, the manifold curvature is represented by a complicated object generalizing the Gauss curvature, which is called today the Riemann curvature tensor. In some sense, Gauss' point of view of a geometry of embeddings (he was also concerned with the embedding in the Euclidean space of non-Euclidean - such as hyperbolic geometries) was somehow bypassed with Riemann's new standpoint of forgetting embeddings for a while, and thinking instead of surfaces intrinsically, with the notion of manifold. It should also be stressed that Riemann's intrinsic approach is the essence of Einstein-Poincaré's new paradigms of physics in the early 20th century.

After Riemann (and Weyl [80]), Whitney was first in 1936 [69,81] to provide a complete formulation of the notion of manifold. His famous result is the following: any $m$-dimensional differentiable manifold can be smoothly embedded in $\mathbb{R}^{2 m}$. By embedding it is intended an injective immersion (an immersion is a differentiable function between manifolds where the derivative has everywhere full rank). Later, the Nash-Kuiper embedding theorem of 1954/55 [43,54], in conjunction with Whitney's result, states that any $m$-dimensional Riemannian manifold $(M, g)$ can be $\mathcal{C}^{1}$-isometrically embedded in an Euclidean space of dimension $2 m+1$ [14]. The importance of this theorem in the history of Mathematics is unvaluable, since it in particular reconciles (Gauss') embeddings- with (Riemann's) intrinsic views.

With this survey, we would like to emphasize the potential of intrinsic approaches in the mathematical modeling of elasto-plastic solids. In particular we begin by identifying the deformation of our body with a metric $g$ and emphasize the role of curvature and torsion in the presence of line-like defects, ultimately leading to plastic effects at the macroscale. Precisely, the notion of incompatibility is at the heart of a new paradigm to describe inelastic effects, since incompatibility is indeed a linearized version of Riemannian curvature, as we will see. The starting point of the theory is to accept that, in the spirit of Riemann, we should attack the modeling problem of elasto-plasticity with an instrinsic approach, that is geometric in nature. Indeed, incompatibility is a physical notion related to non-smooth, in a certain sense singular deformations in the following acception: quoting Cartan $[12]^{1}$, "the Riemannian space is for us an ensemble of small pieces of Euclidean space, lying however to a certain degree amorphously", while about crystals where dislocation form, Kondo [39] suggests that "the defective crystal is, by contrast [with respect to the above by him given definition of perfect crystal], an aggregation of an immense number of small pieces of perfect crystals (i.e. small pieces of the defective crystal brought to their natural state in which the atoms are arranged on the regular positions of the perfect crystal) that cannot be connected with one other so as to form a finite lump of perfect crystals as an organic unity". In the sequel, we develop these ideas, and in particular we set up a precise mathematical understanding of these concepts.

\section{Curvature in nonlinear Elasticity}

Consider now the manifold to be an open subset $\Omega$ of $\mathbb{R}^{3}$, and consider a symmetric positive definite tensor $g_{i j}$. The question raised is the following: under which conditions is $(\Omega, g)$ flat, that is, there exists an immersion $\Theta: \Omega \rightarrow \mathbb{R}^{3}$ such that $g$ is the metric tensor of the open set $\hat{\Omega}:=\Theta(\Omega)$ ? (Note that manifold and embedding spaces have here the same dimension, as opposed to Whitney-Nash theorems). To answer this question, let us first assume that such an immersion

\footnotetext{
${ }^{1}$ This was in essence Riemann's definition of a manifold: each point is in a neigborhood ressembling a distorted Euclidean space.
} 
exists. Then, by definition, it exists a local frame $\left\{\underline{g}_{i}\right\}_{1 \leq i \leq 3}$ with $\underline{g}_{i}:=\partial_{i} \Theta$ such that $g_{i j}:=\underline{g}_{i} \cdot \underline{g}_{j}$. Then the following theorem holds: necessarily, the Riemann curvature tensor Riem $_{g}$ associated to $g_{i j}$ vanishes in $\Omega$. Of particular interest is the reciprocal statement: given an open, connected and simply connected set $\Omega$ and an arbitrary metric $C:=g_{i j}$, if $\operatorname{Riem}_{g}=0$, then there exists $\Theta \in \mathcal{C}^{3}\left(\Omega ; \mathbb{R}^{3}\right)$ such that $C=(\nabla \Theta)^{T} \nabla \Theta$, i.e., $g_{i j}=\partial_{i} \Theta \cdot \partial_{j} \Theta$. Proof of these theorems and their variants can be found in [14].

In elasticity $\Omega$ is the reference body, $\hat{\Omega}$ is its deformation, $F:=D \Theta=\nabla \Theta$ is the associated deformation tensor, and $C$ the right Cauchy-Green tensor. As a matter of fact, a restatement of this theorem reads: given a reference body $\Omega$ and an intrinsic measure $C$ of its deformation (in elasticity $C$ is known to account for local stretch and rotations, [67]), when does it exist a tensor $F$ such that $C=F^{T} F$ also satisfying Curl $F=0$ ? Indeed, it is well known (HelmholtzWeyl-de Rahm decompositon-type results, also known, in the sense of distributions, as Poincaré's Lemma [14], [40]) that

$$
\text { Curl } F=0 \quad \Leftrightarrow \quad F=D \phi \text { for some } \phi,
$$

with Curl and $D$ intended in the sense of distributions. Therefore we can write that given $\Omega$ open connected and simply connected, and provided a symmetric, positive definite tensor $C$ one has:

$$
\exists F: \text { Curl } F=0, C=F^{T} F \quad \Leftrightarrow \quad \operatorname{Riem}_{C}=0 .
$$

In the presence of line-like defects such as Volterra dislocations [35], we are typically faced to the following issue: we have an elastic body $\Omega$ with a dislocation loop $\mathcal{L}$ and we assume that we have the means for determining at any point of $\Omega \backslash \mathcal{L}$ the stretch and rotation, in other words we are given a metric tensor $C$. It turns out that we are only able to construct such a deformation $F$ as in (2.1) and (2.2) in $\Omega \backslash \Pi_{\mathcal{L}}$ where $\Pi_{\mathcal{L}}$ is a surface containing $\mathcal{L}$ and dividing $\Omega$ into two subdomains $\Omega^{+}$and $\Omega^{-}$[59]. Let $S_{\mathcal{L}} \subset \Pi_{\mathcal{L}}$ be the surface enclosed by the loop, i.e. $\partial S_{\mathcal{L}}=\mathcal{L}$. Therefore, above and below $\Pi_{\mathcal{L}}$ there exists $\phi^{+}$and $\phi^{-}$, respectively, such that $C=F^{T} F$ with $F=D \phi^{ \pm}$in $\Omega^{ \pm}$. However, it turns out that by the Volterra construction, there is constant jump on $S_{\mathcal{L}}$ that we denote by $b$, the Burgers vector of $\mathcal{L}$. Specifically, $\phi:=\mathrm{Id}+u$ exists globally in $\Omega$ by means of a function of bounded variation $u$, i.e. whose distributional derivative satisfies $D u=\nabla u+b \otimes \nu_{S_{\mathcal{L}}} d \mathcal{H}_{\left\lfloor S_{\mathcal{L}}\right.}^{2}$ where $\nu_{S_{\mathcal{L}}}$ is the unit normal to $S_{\mathcal{L}}$. Therefore, in $\Omega \backslash \mathcal{L}$ one may define the deformation tensor as $F=\mathbb{I}+\nabla u\left(=D \phi^{ \pm}\right.$in $\left.\Omega \backslash S_{\mathcal{L}}\right)$ such that $C=(\mathbb{I}+\nabla u)^{T}(\mathbb{I}+\nabla u)$. However in $\mathcal{L}$ this representation fails, and hence the aforementioned approach holds piecewise. Nonetheless, something can be said at $\mathcal{L}$, namely by use of Stokes theorem [59], one finds

$$
-\operatorname{Curl} F=-\operatorname{Curl} \nabla u=\Lambda_{\mathcal{L}}^{T}:=b \otimes \tau_{\mathcal{L}} d \mathcal{H}_{L \mathcal{L}}^{1},
$$

where $\Lambda_{\mathcal{L}}$ is called the dislocation density. This reasoning can be generalized for $\mathcal{L}$ a countable union of rectifiable dislocations. This leads us to the following conclusion: the dislocations prevent the deformation to be Euclidean and indeed no global embedding exists. We refer to [82] for a recent contribution to the topic.

The mathematical nature of the displacement field has been clarified in [60,61]: given the set of dislocations $\mathcal{L}$, an integral current (i.e., the generalization of a closed Lipschitz loop) and a deformation tensor $F$ satisfying (2.3), there exists $u \in W^{1, p}\left(\Omega, \mathbb{T}^{3}\right) \cap S B V\left(\Omega, \mathbb{R}^{3}\right) \cap \mathcal{C}{ }^{\infty}\left(\Omega \backslash \mathcal{L}, \mathbb{T}^{3}\right)$ such that the following holds in the sense of distributions:

$$
\text { Div } \nabla u=\Delta u=\operatorname{Div} F \text { and }-\operatorname{Curl} \nabla u=b \otimes \tau_{\mathcal{L}} d \mathcal{H}^{1}{ }_{L}=-\operatorname{Curl} F \text {. }
$$

The crucial point to note is that the displacement field can be seen in various ways: either as a multi-valued (i.e., in the three-dimensional distorted flat torus $\mathbb{T}^{3}$ for a normalized Burgers vector, meaning that each component $u_{i}$ is identified with $u_{i}+2 \pi \mathbb{Z}$ ) Sobolev vector field (note that $(2.3$ ) imposes that $1 \leq p<2$ ), or as a special function of bounded variation, whereby it exhibits a jump on a surface $S_{\mathcal{L}}$ which is not unique (it must be a Lipschitz surface with $\mathcal{L}$ as boundary). Moreover away from $\mathcal{L}$ the displacement is smooth, however multivalued. We can write

Dislocation in nonlinear elastic bodies $\Rightarrow \operatorname{Curl} F \neq 0$ and $\exists u \in \mathbb{T}^{3}$ s.t. $F=\nabla u$. 


\section{INCOMPATIBILITY IN LINEARIZED ELASTICITY AND PATH INTEGRAL FORMULAE}

Linearized elasticity is obtained by neglecting the quadratic terms in $C=(\mathbb{I}+\nabla u)^{T}(\mathbb{I}+\nabla u)$, that is, we consider a metric $g(u)$ defined as $g_{i j}=\delta_{i j}+2 e_{i j}(u)$ where $e(u)=\nabla^{S} u:=\frac{1}{2}\left(\nabla^{T} u+\nabla u\right)$. Let us consider Riem $e_{e}$ the associated Riemann curvature tensor. It was proved in [46, Proposition 3.11] that

$$
\left(\operatorname{Riem}_{e}\right)_{i j k l}=\epsilon_{i j m} \epsilon_{k l n}(\text { inc } e)_{m n}+\text { h.o.t., }
$$

where inc is the symbol standing for the incompatibility operator, writing in Cartesian coordinates as

$$
\text { inc } e:=\text { Curl } \mathrm{Curl}^{T} e \text {. }
$$

In the above, $\epsilon$ is the Levi-Civita symbol, and the Curl of a tensor is calculated row-wise, hence for a symmetric tensor inc takes the Curl column-wise then row-wise. The equation inc $e=0$ is equivalent to the Saint-Venant compatibility conditions recalled and discussed thereafter. Pioneer contributions linking compatibility conditions and the Riemann curvature tensor can be found in $[27,63]$. We also define the Frank tensor as

$$
\mathcal{F}(e):=\operatorname{Curl}^{T} e .
$$

Let us recall now the problem of reconstructing a displacement from a given symmetric tensor ${ }^{2}$. In linearized elasticity, if all the functions involved are smooth enough, the displacement field $u$ turns out to be completely defined in terms of the linearized strain tensor $e$ by an explicit recursive integral formula. Let $e \in C^{\infty}\left(\Omega, \mathbb{M}^{3}\right)$ be a symmetric tensor field in $\Omega$. Let us fix $x_{0}, x \in \Omega$, and let $\gamma \in C^{1}([0,1], \Omega)$ be a curve in $\Omega$ such that $\gamma(0)=x_{0}$ and $\gamma(1)=x$. We define the following quantities:

$$
w_{i}(x ; \gamma):=w_{i}\left(x_{0}\right)+\int_{\gamma} \epsilon_{i p n} \partial_{p} e_{m n}(y) \mathrm{d} y_{m}, \quad u_{i}(x ; \gamma):=u_{i}\left(x_{0}\right)+\int_{\gamma}\left(e_{i l}(y)-\epsilon_{i l k} w_{k}(y)\right) d y_{l}
$$

Note that $\mathcal{F}_{i m}=\epsilon_{i p n} \partial_{p} e_{m n}$, thence the name Frank tensor, since its integral on a closed curve $\gamma$ making one loop around the dislocation $\mathcal{L}$ provides the jump of the rotation vector $w$, classically known as the Frank tensor. The quantities $w(x)$ and $u(x)$ defined in (3.4) do a-priori depend on the choice of the path from $x_{0}$ to $x$. In such a case the quantities $w$ and $u$ define two $C^{\infty}$ functions on $\Omega$ that will be called the multi-valued rotation and displacement vectors associated to the strain $e$, respectively (see [75] for the exact meaning of multivaluedness in this context). However, if one has inc $e=0$ then $u$ and $w$ are single-valued fields, i.e., are unambiguously defined. Thus, in particular in (3.4), one can use the notation $\int_{\gamma}=\int_{x_{0}}^{x}$ to mean that the integral is path independent. In order to prove this fact, we compute the jump of $w$ and $u$ between two arbitrary curves with the same endpoints, namely $\gamma$ and $\tilde{\gamma}$, and observe that this quantity is zero if the incompatibility tensor vanishes. These are exactly the well-known Saint-Venant compatibility relations [14]. The rotation and displacement jumps at $x$ are defined as

$$
\llbracket w_{i} \rrbracket=w_{i}(x ; \gamma)-w_{i}(x ; \tilde{\gamma}), \quad \llbracket u_{i} \rrbracket=u_{i}(x ; \gamma)-u_{i}(x ; \tilde{\gamma}),
$$

respectively, and hence depend on the chosen closed path $\gamma-\tilde{\gamma}$ at $x$. Let $\Omega \subseteq \mathbb{R}^{3}$ be a simplyconnected domain, let $x_{0} \in \Omega$ be prescribed, and let $w, u \in C^{\infty}\left(\Omega, \mathbb{R}^{3}\right)$ be the functions defined in (3.4). Then the following formulae hold:

$$
\llbracket w_{i} \rrbracket=\int_{S}(\operatorname{inc} e(y))_{i m} \mathrm{~d} S_{m}(y), \quad \llbracket u_{i} \rrbracket=\int_{S}\left(y_{m}-x_{m}\right) \epsilon_{i m k}(\text { inc } e(y))_{q k} \mathrm{~d} S_{q}(y),
$$

\footnotetext{
${ }^{2}$ The history of this construction roots in the end of the 19 th century. We have identified by chronological order the following relevant contributions: Kirchhoff in 1876 [37], Beltrami in 1886 [9], Volterra in 1887 [76] (see also [77,78]), Love in 1892 [45], Michell in 1899 [48], Cesàro in 1906 [13] and the Cosserat brothers in 1909 [16]. The first rigourous proof must be assigned to Beltrami. According to Love, though, the bulk compatibility conditions should be credited to Barré de Saint-Venant in 1864 [7].
} 
for all $x \in \Omega$, and where $S$ is a surface enclosed by the the closed path $\gamma-\tilde{\gamma}$. In particular,

$$
\text { inc } e=0 \Rightarrow \llbracket w_{i} \rrbracket=\llbracket u_{i} \rrbracket=0 \text { for every } x \text { and } S \text {. }
$$

Thus, given the tensors $e$ and $\mathcal{F}(e)=\operatorname{Curl}^{T} e$, and as a consequence of inc $e=0$, the vector fields $w$ and $u$ are univoquely defined in (3.4). We refer to [46, Proposition 2.2, Corollary 2.4] for a proof. Moreover, the following classical quantities can be introduced: (i) $e_{i j}:=\frac{1}{2}\left(\partial_{j} u_{i}+\partial_{i} u_{j}\right)$ is the linearized strain tensor (i.e., the linear part of Green St-Venant tensor $C_{i j}=e_{i j}+\partial_{i} u_{k} \partial_{k} u_{i}$ ); (ii) $\omega_{i j}:=\frac{1}{2}\left(\partial_{j} u_{i}-\partial_{i} u_{j}\right)$ is said rotation tensor, with $w_{i}:=\frac{1}{2} \epsilon_{i j k} \omega_{k j}$ the rotation vector.

Therefore, the linearized counterpart of (2.5) reads by (3.6),

$$
\text { inc } e \neq 0 \Rightarrow \exists u, w \text { multiple-valued fields, s.t. } e=\nabla^{S} u \text {. }
$$

In particular, this happens if, given a dislocation loop $L, \gamma-\tilde{\gamma}$ is a curve making one or more loops around $L$. The multiplicity is precisely the number of loops made, while the jump of $u$ is the Burgers vector $b$ (and of $w$, the Frank tensor $\Omega$ ).

\section{The Legacy of EkKehart Kröner: the GeOMEtry of a CRYSTAL With Dislocations}

Given the dislocation density $\Lambda_{\mathcal{L}}$ we have seen that there exists a map $\varphi \in W^{1, p}\left(\Omega, \mathbb{T}^{3}\right), 1 \leq$ $p<2$ such that $-\operatorname{Curl} \nabla \varphi=\Lambda_{\mathcal{L}}^{T}$ with $\operatorname{Div} \nabla \varphi=0$ in $\Omega$, and $(\nabla \varphi) N=0$ on $\partial \Omega$. The first equality stems directly from the Stokes theorem and from the property $\llbracket \varphi \rrbracket=b$ on any enclosing surface $S_{\mathcal{L}}$ [60]. In the same spirit [73], one can find a displacement field $u \in W^{1, p}\left(\Omega, \mathbb{T}^{3}\right), \quad 1 \leq p<2$ satisfying $-\operatorname{Div}\left(\mathbb{A} \nabla^{S} u\right)=f$ and $-\operatorname{Curl} \nabla u=\Lambda_{\mathcal{L}}^{T}$ in $\Omega,\left(\mathbb{A} \nabla^{S} u\right) N=g$ on $\partial \Omega$ and satisfying $\llbracket u \rrbracket=b$ and $\llbracket \mathbb{A} \nabla^{S} u \rrbracket=0$ on $\Omega \cap S_{\mathcal{L}}$. Moreover, it is deduced in [73] that the following expression holds in the sense of distributions:

$$
\text { inc } e(u)=\operatorname{inc} \nabla^{S} u=\operatorname{Curl}\left(\Lambda_{\mathcal{L}}-\frac{\mathbb{I}}{2} \operatorname{tr} \Lambda_{\mathcal{L}}\right) \quad \text { in } \Omega .
$$

This establishes at the mesoscopic scale the famous macroscopic Kröner formula relating elastic strain incompatibility and dislocation density.

4.1. The geometric approach at the macroscale. At the mesoscale we have seen that the dislocation density reads $\Lambda_{\mathcal{L}}=\tau \otimes b d \mathcal{H}_{\perp \mathcal{L}}^{1}$ and represents the quantity of Burgers vector per unit area (as the density measure $d \mathcal{H}_{\lfloor\mathcal{L}}^{1}$ has the dimension of an inverse area). Let $\mathcal{S}$ be a small surface in $\Omega$ with unit normal $n$ and define the cylinder $\mathcal{V}_{\epsilon}=\{x+t n, x \in \mathcal{S},-\epsilon \leq t \leq \epsilon\}$. Consider a family $\mathcal{L}$ of $N$ parallel mesoscopic dislocations with Burgers vector $b$ and orientation $\tau=n$. One has

$$
\frac{1}{2 \epsilon} \int_{\mathcal{V}_{\epsilon}} \Lambda_{\mathcal{L}}^{T} n d V=N b
$$

This corresponds to the definition of dislocation density as used by practitioners and lead Kröner [41] to define the macroscopic Burgers vector of a surface $\mathcal{S} \subset \Omega$ as

$$
B(\mathcal{S}):=\int_{\mathcal{S}} \Lambda^{T} d S
$$

a macroscopic quantity related to the number of dislocation lines crossing $\mathcal{S}$, with $d S$ the oriented area element, and where $\Lambda$ is the assumed smooth macroscopic dislocation density. Moreover, one defines the contortion tensor ${ }^{3}$ as $\kappa=\Lambda-\frac{\mathbb{I}}{2} \operatorname{tr} \Lambda$. Recalling (3.1), there is a direct link between intrinsic curvature and dislocation density, since the macroscopic expression of Kröner's formula (see $[41,71]$ ) reads

$$
\text { inc } \varepsilon=\operatorname{Curl} \kappa \text {. }
$$

Further, at the macroscale we are given a smooth linearized strain $\varepsilon$, and consider the elastic metric

$$
g_{i j}:=\delta_{i j}+\varepsilon_{i j} .
$$

\footnotetext{
${ }^{3}$ This object has a geometrical meaning in non-Riemannian manifolds, see e.g. [62], that we will not detail here.
} 
So far, we have introduced a metric and an intrinsic curvature related to the presence of dislocations. Yet an important notion is missing: that of connection. The connection in geometry is a notion that permits a comparison between the local geometry at one point and the local geometry at another point. It is thus related to the differentiation of tensor fields and indeed it is well-known [19] that a "good notion" of gradient on a manifold is induced by the choice of the connection $\Gamma$ (also known as the Christoffel symbols). Denoting this gradient by $\nabla_{\Gamma}$, one can introduce the notion of parallel transport along a curve: it is said that a vector $v$ is parallely transported along $\gamma(t)$ if $\dot{\gamma} \cdot \nabla_{\Gamma} v=0$. It is said that a connection is compatible with the metric if $\nabla_{\Gamma} g=0$ : in this case two vector fields $v, w$ parallely transported along a curve have the property that their scalar product $g(v, w)$ is constant. Further, in case of compatible symmetric connections, and in this case only, the Christoffel symbols write as (see [19, Theorem 29.3.2])

$$
\Gamma_{i j}^{k}=\frac{1}{2} g^{k l}\left(\partial_{i} g_{l j}+\partial_{j} g_{i l}+\partial_{l} g_{i j}\right) \text {. }
$$

This connection is termed Riemannian or Levi-Civita after the name of the Italian mathematician Tullio Levi-Civita (1873-1943). As a matter of fact, a manifold is said Riemannian if it is endowed with both a Riemannian metric and a Riemannian connection (see, e.g., [25]) and, for us, a nonRiemannian manifold means that the connection need not be symmetric and compatible. Note that this latter expression of $\Gamma_{i j}^{k}$ is the unique symmetric connection compatible with the metric $g:=\mathbb{I}+\varepsilon$ and is that considered to obtain (3.1) from the Riemann curvature tensor Riem $\varepsilon$ associated to it. In the sequel this metric connection will be denoted ${ }^{4}$ by $\Gamma^{B}$.

Now, for non-Riemannian connections, the crucial notion is that of connection's torsion, defined as the tensor $T$ writing component-wise as

$$
T_{i j}^{k}=\Gamma_{[i j]}^{k}:=\frac{1}{2}\left(\Gamma_{i j}^{k}-\Gamma_{j i}^{k}\right) .
$$

4.2. Parallel displacement and curvature. The role of parallel displacement in a perfect crystal is emphasized by Kröner as he says [42]: "when a lattice vector is parallely displaced using $\Gamma^{B}$ along itself, say 1000 times, then its start and goal are separated by 1000 atomic spacings, as measured by $g$. Because the result of the measurement by parallel displacement and by counting lattice steps is the same, we say that the space is metric with respect to the connection $\left[\Gamma^{B}\right] . "$ Let us translate Kröner's words in formulae. Consider a closed loop $C=\{\gamma(t): t \in[0,1]\}$, with $\dot{\gamma}(t)$ the unit tangent vector. Let us transport a lattice vector $e$ along $C$. In the absence of defects one has an Euclidean connection and hence $e$ is parallely transported along $C$, i.e. $\nabla_{; C} e=\dot{\gamma} \nabla e=\dot{\gamma}^{k} \partial_{k} e=0$. Assume now the presence of dislocations along $C$. We know that the manifold has some curvature and consider in a first step the Levi-Civita connection $\Gamma=\Gamma^{B}$ associated to $g$. To transport the contravariant vector $e$ we need to compute its covariant derivative along $C$, namely $\left(\nabla_{; C} e\right)^{i}:=\dot{\gamma}^{k}\left(\partial_{k} e^{i}+\Gamma_{j k}^{i} e^{j}\right)$ (cf. [19, §28.2., Eq. (23)]). Therefore the instantaneous deviation of $e$ due to dislocations is given by

$$
\left(\nabla_{; C} e\right)^{i}=\Gamma_{j k}^{i} e^{j} \dot{\gamma}^{k}
$$

In linearized elasticity it is assumed $g_{k l}=\delta_{k l}+2 \varepsilon_{k l}$ and thus lowering and raising the indices can be considered indifferently, to the first order. So, from $\Gamma_{k ; j i}:=g_{k l} \Gamma_{j i}^{l}$, the deviation of $e^{i}$ in a time interval $d t$ writes as $\Gamma_{i ; j k} e_{j} d x_{k}+$ h.o.t., with $d x^{k}:=\dot{\gamma}^{k} d t$. Let us compute the total amount of deviation on a closed loop $C$ up to the first order, that is, we calculate $\int_{C} \Gamma_{i ; j k} d x_{k}$ that by Stokes theorem rewrites as $\int_{S_{C}} \epsilon_{m q k} \partial_{q} \Gamma_{i ; j k} d S_{m}$, with $S_{C}$ a surface enclosed by $C$. The covariant derivative of $\Gamma_{i ; j k}$ reads (cf. [19, Eq. (32)])

$$
\nabla_{q} \Gamma_{i ; j k}=\partial_{q} \Gamma_{i ; j k}-\left(\Gamma_{i ; p k} \Gamma_{p ; j q}+\Gamma_{i ; p q} \Gamma_{p ; j k}\right)-\Gamma_{i ; j p} \Gamma_{p ; k q} .
$$

By the symmetry in $q$ and $k$ of the term inside the parenthesis and of the last term, this yields in the absence of torsion

$$
\epsilon_{m q k} \partial_{q} \Gamma_{i ; j k}=\epsilon_{m q k} \nabla_{q} \Gamma_{i ; j k}
$$

${ }^{4}$ Subscript $B$ stands for the Bravais crystal, cf. [70]. 
Now, let $\left\{\mathbf{a}_{\mathbf{i}}\right\}$ denote an orthonormal basis, and recall that $\Gamma_{j k}^{i}=\left(\nabla_{k} \mathbf{a}_{j}\right)^{i}$ (cf. [19, Eq. (33)]). Thus, by a property of the Riemann curvature tensor (or a definition, see [19, Theorem 30.1.1])), one has

$$
\begin{aligned}
\int_{C} \Gamma_{i ; j k}^{B} d x_{k}=\int_{S_{C}} \epsilon_{m q k} \nabla_{q}\left(\nabla_{k} \mathbf{a}_{j}\right)_{i} d S_{m}+\text { h.o.t. } & =\int_{S_{C}} \frac{1}{2} \epsilon_{m q k}\left(\operatorname{Riem}_{g}^{B}\right)_{i ; p k q}\left(\mathbf{a}_{j}\right)_{p} d S_{m}+\text { h.o.t. } \\
& =\int_{S_{C}} \frac{1}{2} \epsilon_{m q k}\left(\operatorname{Riem}_{g}^{B}\right)_{i ; j k q} d S_{m}+\text { h.o.t.. }
\end{aligned}
$$

By virtue of (3.1)-(3.3) this yields

$$
-2 \int_{C} \Gamma_{i ; j k}^{B} d x_{k}=\epsilon_{i j p} \int_{S_{C}}(\text { inc } \varepsilon)_{p m} d S_{m}+\text { h.o.t. }=\epsilon_{i j p} \int_{S_{C}} \epsilon_{m q l} \partial_{q}(\mathcal{F}(\varepsilon))_{p l} d S_{m}+\text { h.o.t., }
$$

that is, by (3.4),

$$
-2 \int_{C} \Gamma_{i ; j k}^{B} d x_{k}=\epsilon_{i j p} \int_{C}(\mathcal{F}(\varepsilon))_{p l} d x_{l}+\text { h.o.t. }=\epsilon_{i j p} \llbracket w_{p} \rrbracket\left(S_{C}\right)+\text { h.o.t.. }
$$

Therefore we see that at the first order, the total deviation of $e^{i}$ around $C$ depends on the rotation jump on the surface, since

$$
\int_{C} \nabla_{k} e_{i} d x_{k}=\frac{1}{2} \epsilon_{i k l} \Omega_{k}\left(S_{C}\right) e_{l}+\text { h.o.t., }
$$

where we have introduced the Frank tensor $\Omega_{k}:=\llbracket w_{k} \rrbracket$. So far we have established the following:

\section{Point view of the external observer:}

- $g$ Riemannian metric, $\Gamma^{B}$ Riemannian connection $\Rightarrow\left(\Omega, g, \Gamma^{B}\right)$ Riemannian manifold.

- Observation 1: $\nabla \Lambda \neq 0, T=T^{B}=0 \Rightarrow \operatorname{Riem}_{g} \neq 0$ :

non-homogeneous dislocation density $\Rightarrow$ crystal manifold with curvature but no torsion.

- Observation 2: $\int_{C} \nabla e \cdot d L=\frac{1}{2} \Omega\left(S_{C}\right) \times e+$ h.o.t. : disclinations $\Rightarrow$ crystal curvature.

In this case the anholonomy is given by the presence of disclinations, where the term "holonomy" 5 of a connection refers to the extent to which parallel transport around closed loops achieves, or fails, to preserve the geometrical data being transported: for instance under a metric connection, the orthogonality of two parallely transported vector fields is preserved.

In this first perspective, of the so-called external observer, the absence of isometric embedding in $\mathbb{R}^{3}$ is translated into the nonvanishing Riemann curvature tensor associated with the LeviCivita metric connection of $g$. Though, one can see that something is missing in this formalism, since a homogeneous (constant) density of dislocations yields a crystal manifold with vanishing curvature, that is, a flat manifold. Moreover a pure dislocation (i.e, with vanishing jump of the rotation tensor) would permit parallel transport of lattice vectors, in contradiction with the nature of the dislocations which is responsible for atomic jumps, hence inducing a geometry that is not Euclidean. Instead, we would like to find a geometry that is specific for crystals with dislocations. This will be achieved by means of a new, non-Riemannian connection, i.e. a connection with torsion. This new perspective requires from the standpoint of physics the introduction of the so-called internal observer, able to determine crystallographic defects. For a recent review on incompatible deformation fields and the Riemann curvature field, we refer to [65].

\footnotetext{
${ }^{5}$ The holonomy group is defined as the set of linear transformations arising from parallel transport along closed loops.
} 
4.3. The non-Riemannian crystal manifold. To emphasize the perspective of the internal observer we define the following geometrical objects:

$\begin{array}{lrl} & T_{i ; k j} & :=\epsilon_{k j q} \Lambda_{q i} \\ \text { DisLOCATION TORSION: } & \Delta \Gamma_{k ; i j}:=-T_{j ; i k}-T_{i ; j k}+T_{k ; j i} \\ \text { CONNECTION CONTORTION: } & \Gamma_{k ; i j}:=\Gamma_{k ; i j}^{B}+\Delta \Gamma_{k ; i j} .\end{array}$

It is easily seen that $\Gamma$ is a connection, since $\Gamma^{B}$ is a connection and $\Delta \Gamma$ is a tensor (by the transformation property of a connection, cf. [19, Eq. (22)]). Moreover the symmetric part of $\Gamma$ namely $\Gamma_{k ;(i j)}$ is a connection and it can be proved [70, Theorem 5.2] that $\Gamma_{k ;[i j]}=T_{k ; i j}$. Denoting the symmetric part of the connection by $\Gamma_{k ; i j}^{\circ}=\Gamma_{k ; i j}^{B}+\Delta \Gamma_{k ;(i j)}$ we have, by the above calculations, one has

$$
\int_{C} \Gamma_{i ; j k}^{\circ} e_{j} d x_{k}=\int_{S_{C}} \frac{1}{2} \epsilon_{m q k}\left(\operatorname{Riem}^{\circ}(\varepsilon)\right)_{i ; j k q} e_{j} d S_{m}+\text { h.o.t. }
$$

where $\operatorname{Riem}^{\circ}(\varepsilon)$ is the Riemann curvature tensor associated to $\Gamma^{\circ}$. Let us now compute the remaining term, namely

$$
\int_{C} \Gamma_{i ;[j k]} e^{j} d x_{k}=\int_{C} T_{i ; j k} e_{j} d x_{k}=\int_{C} \Lambda_{p i} d S_{p},
$$

where we have introduced the surface element $d S_{p}=\epsilon_{p j k} e_{j} d x_{k}$. The deviation of the second term is just simply the integral on $C$ of the macrosopic Burgers vector $B_{i}(d S)$, that is

$$
\int_{C} \Gamma_{i ;[j k]} e^{j} d x_{k}=\int_{C}(n \cdot \tau) B_{i}(d S)
$$

with $d S_{p}=n_{p} d S$.

Thus we see that provided the metric and the connection of the internal observer, the lattice vector is deviated: the first source of deviation is the Riemannian curvature, whereas the second is the connection's torsion. Note that this connection might also be not metric, i.e., if $\nabla g \neq 0$. The metric tensor is considered to model point defects in crystals, but this is beyond the scope of this survey (cf [83]).

4.4. Internal and external observers. These are physical concepts related to fictitious thought experiments. The external observer is only able to make experiments from the outside, namely it measures fiber elongations and/or rotations and hence it measures a deformation $\varepsilon$ that provides the geometer with a metric $g$. For the external observer the utmost geometrical quantity available is the Riemannian curvature as derived from the metric connection $\Gamma^{B}$. On the contrary, the internal observer acts at another scale. It is able to recognize crystallographic direction and count atomic steps, hence can measure a density of dislocations. Thus torsion is available to the internal observer, but not to the external. It is only a combined view that provides a complete geometrical picture of the dislocated crystal, thence described as a non-Riemannian manifold, summarized as follows:

\section{Combined views of the internal and external observers:}

- $g$ Riemannian metric, $\Gamma$ non-Riemannian connection $\Rightarrow(\Omega, g, \Gamma)$ non-Riemannian manifold.

- Property: $(\Lambda \neq 0 \Rightarrow T \neq 0)$ and $\left(\nabla \Lambda \neq 0 \Rightarrow \operatorname{Riem}_{\Gamma} \neq 0\right)$ :

non-homogeneous dislocation density $\Rightarrow$ crystal manifold with curvature and torsion;

homogeneous dislocation density $\Rightarrow$ crystal manifold with torsion.

- Anholonomy by displacement and rotation jumps (i.e., dislocations and disclinations):

$\int_{C} \nabla_{k} e_{i} d x_{k}=\int_{C}(n \cdot \tau) B_{i}(d S)+\epsilon_{i k l} \Omega_{k}\left(S_{C}\right) e_{l}+\cdots$. 
We note that if one restricts to the first order then it holds true that $\int_{C} \nabla_{k} e_{i} d x_{k}=\int_{S_{C}} \frac{1}{2} \epsilon_{m q k}$ $\left(\operatorname{Riem}_{\Gamma}(\varepsilon)\right)_{i ; j k q} e_{j} d S_{m}$ with $\operatorname{Riem}_{\Gamma}$ the Riemann curvature of the connection $\Gamma$ defined as a sum with $\Gamma^{B}$ in such a way that $\operatorname{Riem}_{\Gamma}=\operatorname{Riem}^{B}+\cdots$ with the remaining terms related to $\Delta \Gamma$. Note also that it is solely Riem $^{B}$ that yields the term $\Omega\left(S_{C}\right) \times e$.

As we see, Kröner's macroscopic framework allows us to come back to the language of geometry, by stressing that the crystal geometry and the physical laws governing defects are inseparable, as is the case in the Einstein's general theory of relativity. We entirely agree with Noll when he writes [55] that "the geometry [must be] the natural outcome, not the first assumption, of the theory" 6 . Many geometrical tools and mathematical theory required for a rigorous description of the dislocated crystal geometry can be found in the landmark papers by Noll [55] and Wang [79], while also pointing out a recent book on continuum mechanics in this spirit [22]. We emphasize that $\Delta \Gamma_{k ;[j i]}$ was also introduced by Noll [55] and called the crystal inhomogeneity tensor.

4.5. Inelastic effects and notion of eigenstrain. The geometric description of a dislocated body has been made so far for static dislocations, that is, at the macroscale for a constant in time dislocation density tensor. We have seen that spatial variation of $\Lambda$ and hence of the contortion tensor $\kappa$ induces a non vanishing Curl $\kappa$ thence a nonzero elastic strain incompatibility inc $\varepsilon$. A further notion introduced by Kröner is the eigenstrain $\bar{\varepsilon}$ satisfying inc $\bar{\varepsilon}=-$ inc $\varepsilon$. Physically it represents the additional strain to recover compatibility, since inc $(\bar{\varepsilon}+\varepsilon)=0$ implies the existence of a vector field $u$ such that $e(u)=\bar{\varepsilon}+\varepsilon$ as related to the so-called Beltrami decomposition of symmetric tensor fields and Saint-Venant conditions [46]. Plasticity is the macroscopic behaviour of a body whose dislocation density tensor varies in time, since dislocation motion is the physical cause of plasticity. The last sections of this survey will be dedicated to the description of a novel model of elasto-plastic bodies based on $\varepsilon$ and inc $\varepsilon$, hence the model variables are $(\varepsilon, \kappa)$, two intrinsic and objective tensors, in an extended sense to be defined later. Before, we intend to recall the intrinsic approach to linearized elasticity.

\section{A GEOMETRIC CONCEPTION OF LINEARIZED ELASTICITY: THE INTRINSIC APPROACH}

5.1. Gauss vs. Riemann in linearized elasticity. In the conventional mathematical treatment of linearized elasticity, the basic model variable is the displacement field $u: \Omega \rightarrow \mathbb{R}^{3}$, with respect to which elastic problems are stated and solved. Further, the linearized strain is introduced in a second step as the symmetric gradient of the displacement field, $\varepsilon=e(u):=\nabla^{S} u$. However, in many computations and experiments, the strain is most naturally the "observable" field, thence becoming the main model variable. In this spirit, the stress might also be considered as a root variable, in the sense that it is a field that is observable, measurable and controllable: by a possibly fictitious thought experiment, the stress is obviously measurable by extracting from the elastic body a small enough volume element and then measuring the Newtonian forces exerted on its facets. Moreover, given the stress tensor, the strain is well defined as soon as a constitutive law is provided, here a linear homogeneous, isothermal and isotropic law: the strain-stress constitutive law reads $\varepsilon=\mathbb{C} \sigma$, with $\mathbb{C}$ the compliance tensor, i.e., fourth-rank (inverse) tensor of elasticity.

However, today most elasticity problems are treated using the displacement as basic model variable, from which the strain is defined by the kinematic relation $\varepsilon=\nabla^{S} u$, thence the stress by a constitutive law. This approach presumably comes from the study of elliptic boundary-value problems, where the elasticity system is most often presented as a vector-valued extension of elliptic equations in divergence form. Moreover, weak and variational formulations are most easily derived by means of the displacement, and show a convenient and elegant way of solving problems in elasticity.

There are nonetheless profound theoretical reasons to refrain from taking the displacement as main model variable. For instance, its possible multi-valuedness, which is not to avoid from a physical standpoint, since multi-valuedness may have a meaning, but which must be addressed

\footnotetext{
${ }^{6}$ As in the Continuous Distribution of Dislocation (CDD) theory of Bilby et al. [10].
} 
in an adequate manner in an appropriate mathematical formalism (see above). Another example is the reference configuration, from which the displacement is defined and which by definition is arbitrary: although natural in finite elasticity, it becomes somehow artificial in linearized elasticity, since Eulerian and Lagrangian representations coincide. Moreover, in elasto-plasticity or for elastic bodies with defects, the stress and defect-free reference configurations might not exist (simultaneously). Hence, to remedy this issue, it is often appealed to "intermediate" reference configurations, from which plastic and elastic deformations are defined, but whose physical as well as mathematical meaning are far from clear. In fact, what is a plastic distortion (i.e., the "plastic" part of the displacement gradient) as long as no constitutive law exist for the rotations (i.e., the skew-symmetric part of the gradient)? Not to mention some models which introduce plastic and elastic displacements, whose physical and mathematical meanings are extremely vague. Further, we should also mention the fact that any rigorous model should in principle be proven independent of the choice the reference configuration. Lastly, in the presence of crystal defects like dislocations the very notion of displacement or velocity is not clearly defined at any scale. For instance, at the atomic scale, bonds can move while atoms remain fixed.

5.2. Ciarlet's intrinsic approach to linearized elasticity. As we have seen, it may happen that because of defects or other incompatibilities, the very notion of a displacement field does not make sense as a conventional single-valued field. Instead, one would like to state the linearelastic problem in terms of the strain $\varepsilon$, which needs not a-priori be taken as a symmetric gradient. For these reasons, the intrinsic approach in linearized elasticity by $\mathrm{Ph}$. Ciarlet and C. Mardare [15] constitutes a major breakthrough in mathematical elasticity, which was able to reconcile in an elegant manner the two aforementioned approaches. In their presentation, the strain is the main model variable in terms of which strong as well as variational formulations are sought. The displacement only appears in a second step if the Riemannian curvature tensor associated to the elastic metric vanishes (see above). In the approach of Ciarlet and Mardare, a differential geometry setting is chosen (see above), where the boundary under analysis is defined by means of smooth enough immersions, from which the curvilinear basis, the metric, the symmetric connection and the curvature tensors are derived. It should be emphasized that such derived curvilinear bases are indeed defined in the body itself as well as on its boundary, but are not mutually orthogonal, and have no particular physical meaning. The main difficulty is the treatment of the boundary conditions, since a condition such as $u_{\mid \Gamma_{0}}=0$ for some $\Gamma_{0} \subset \partial \Omega$ is not easily translated to a boundary condition on the deformation tensor.

Let $\varepsilon$ be a compatible strain tensor, i.e., by Saint-Venant theorem (see below), there exists a displacement field $u$ such that $\varepsilon=e(u)=\nabla^{S} u$. Let $\varepsilon_{T}$ be the tangential strain (i.e. the projection of $e$ perpendicularly to the normal to the boundary) $\partial \Omega$. Let $\gamma^{\sharp}:=\varepsilon_{T \mid \partial \Omega}$ be the linearized changeof-metric and $\rho^{\sharp}$ the linearized change-of-curvature tensors as introduced by Ciarlet-Mardare in [15]. Let $\Gamma \subset \partial \Omega$ be a connected relatively open set, and let $\mathcal{R}(\Gamma)$ be the set of rigid motions (i.e., rototranslations) on $\Gamma$. Their main theorem states the following:

Theorem 5.1 (Ciarlet-Mardare [15, Theorem 6.1]). Let $u \in H^{1}(\Omega)$. Let either

(i) $u_{\mid \Gamma}=0$

(ii) $\bar{\gamma}^{\sharp}(e)=\bar{\rho}^{\sharp}(e)=0$

(iii) $u_{\mid \Gamma} \in \mathcal{R}(\Gamma)$.

One has $(i) \Rightarrow($ ii $) \Rightarrow($ iii $)$, where $\bar{\gamma}^{\sharp}$ and $\bar{\rho}^{\sharp}$ are suitable extensions of $\gamma^{\sharp}$ and $\rho^{\sharp}$.

To give a more physical understanding of the linearized change-of-curvature tensor, we need to anticipate the specific trace operators $\mathcal{T}_{0}$ and $\mathcal{T}_{1}$ that have been introduced in [3]. They have been obtained through a Green-like formula (see below Theorem 8.3 for details) where $T$ and $\eta$ are smooth enough symmetric tensors,

$$
\int_{\Omega} T \cdot \operatorname{inc} \eta d x=\int_{\Omega} \operatorname{inc} T \cdot \eta d x+\int_{\partial \Omega} \mathcal{T}_{1}(T) \cdot \eta d S(x)+\int_{\partial \Omega} \mathcal{T}_{0}(T) \cdot \partial_{N} \eta d S(x) .
$$


It has been proved in [2, Lemma 2.11] that that

$$
\text { (i) } \Rightarrow(\text { ii })^{\prime} \quad \varepsilon_{T}=\mathcal{T}_{0}(\varepsilon)=0 \text { and } \mathcal{T}_{1}(\varepsilon)=0 \text { on } \Gamma,
$$

and that, for $\Gamma=\partial \Omega$ (see [2, Proposition 2.19]),

$$
\text { (ii) }{ }^{\prime} \Rightarrow \text { (iii). }
$$

Note that the rigid displacement is set to zero as soon as the normal components of $\varepsilon$, i.e., $\varepsilon-\varepsilon_{T}$ vanish. The link with the Frank tensor is the following:

$$
\varepsilon=0 \text { on } \Gamma \Rightarrow\left(\operatorname{Curl}^{T} \varepsilon \times N=0 \Leftrightarrow \mathcal{T}_{1}(\varepsilon)=0 \text { on } \Gamma\right),
$$

with $N$ the outer normal to $\partial \Omega$. In this case either conditions inc $\varepsilon=0$ in $\Omega$ together with $\varepsilon=\operatorname{Curl}^{T} \varepsilon \times N=0$ or $\varepsilon=\mathcal{T}_{1}(\varepsilon)=0$ on $\Gamma$ implies that $\varepsilon=\nabla^{S} v$ with $v=0$ on $\Gamma$ (since in this case $\left.\bar{\rho}^{\sharp}(\varepsilon)=0[72]\right)$. Thus, the intrinsic elasticity system writes in strong form as

$$
\left\{\begin{array}{ccc}
-\operatorname{div}\left(\mathbb{C}^{-1} \varepsilon\right)=f & \text { in } & \Omega \\
\operatorname{inc} \varepsilon=0 & \text { in } & \Omega \\
\mathcal{T}_{0}(\varepsilon)=\mathcal{T}_{1}(\varepsilon)=0 & \text { on } & \Gamma \\
\left(\mathbb{C}^{-1} \varepsilon\right) N=g & \text { on } & \partial \Omega \backslash \Gamma
\end{array},\right.
$$

where the traces are intended in a weak sense, and with $f$ and $g$ the volume and surface loads, respectively. The corresponding intrinsic variational formulation reads

$$
\inf _{\substack{\text { inc } e=0 \\ \mathcal{T}_{0}(e)=\mathcal{T}_{1}(e)=0 \text { on } \Gamma}} \int_{\Omega}\left(\frac{1}{2} \mathbb{C}^{-1} e-\mathbb{K}\right) \cdot e d x,
$$

where $\mathbb{K}$ is a tensor of external forces satisfying

$$
\left\{\begin{array}{ccc}
-\operatorname{div} \mathbb{K}=f & \text { in } & \Omega \\
\mathbb{K} N=g & \text { on } & \partial \Omega \backslash \Gamma
\end{array} .\right.
$$

\section{The Classical ROUTE TO Plasticity}

After the pioneer works by Coulomb (1773), the theory of plasticity finds its roots in the mid 19th and early 20th centuries by experimentalists (Tresca, 1864) and engineers/mathematicians/physicists (e.g., Saint-Venant ${ }^{7}, 1870$, Von Mises, 1913, Prandtl, 1924), and was later developed by Drucker (1947) and Hill (1950). Importants works were achieved by Taylor and Orowan (1934) putting the spot light on the link between plasticity and dislocation motion.

6.1. The mathematical approaches: two perspectives. The mathematical literature starts with Hodge and Prager around 1950 who were first to understand plasticity in modern mathematical terms, proposing a variational formulation in terms of the stress rate and based on the principle of virtual power, while in parallel Greenberg [28] proposed a variational formulation in terms of the velocities. Thus we see that historically the two approaches of strain vs. displacement-based elasto-plasticity models have already coexisted since the beginning. This is a key aspect of our approach, which is an intrinsic model, i.e., considering the elastic strain as basic model variable, in the sense of the geometers mentioned above, of Prager, and recently (notably after decades of displacement-based approach) of Ciarlet and coauthors [14,15]. The philosophical standpoint can be recast in view of the aformentioned history as follows:

\section{Riemann's view}

Intrinsic models

No reference configuration

Strain/strain rate-based approaches

\section{Gauss' view}

Embeddings models

Reference configurations

Displacement/velocity-based approaches.

\footnotetext{
${ }^{7} \mathrm{~A}$ name that we have encountered already above an which is also related to the incompatibility operator, see below.
} 
Let us now describe the main ingredients of the conventional, historical approach, which, we recall, has proven so far in complete agreement with the observations. It is hence an excellent model for all practical purposes. In the general framework of thermodynamics, one postulates the existence of a dissipation potential which provides the evolution laws for plastic deformation (the so-called flow rules) and possible other internal variables. This part of the theory is strongly linked with convex analysis and thus was fostered in the 60ies by the remarkable works by Rockafellar and Moreau [52]. In the end of the 70ies and in the 80ies, Strang and Temam [68] and their collaborators introduced a new functional space to describe plasticity, the space of bounded deformations, whose main feature is to allow the strain to have a regular part and a measure part, thus well-suited to modeling discontinuous phenomena, such as plastic slip on part of the boundary, or shear band formation in perfect plasticity. This theory yielded on the one hand a first rigorous mathematical formulation (i.e., with proofs of existence, etc., see the excellent textbook by Han and Reddy [32]), and on the other hand gave rise to performent numerical schemes. Summarizing, the conventional approach to elasto-plasticity is based on

(i) the velocities (or the displacement field) as first model variable,

(ii) a postulated decomposition in elastic and plastic parts of the total compatible strain, where each part is assumed to model distinct sub-scale phenomena (elastic deformation means variation in inter-atomic distance, whereas plastic deformation is a macroscopic manifestation of the modification of inter-atomic bonds, where dislocations play a role),

(iii) separate constitutive laws for the elastic and plastic strains,

(iv) a convex elastic domain (whose boundary is the so-called yield surface), which is a sufficient condition in order to satisfy the 2nd principle of thermodynamics and further permit the use of convex calculus.

First existence results for linear elasticity/perfect plasticity were provided around 1980 by Johnson [36], Suquet [66], by means of visco-plastic approximations (see also Anzelotti, Giaquinta, Luckhaus $[5,6]$, Hardt and Kinderlehrer [33]). About 20 years later, a quasi-static evolutionary variational formulation was sucessfully proposed by Mielke and coauthors (Mainik, Roubíček, Stefanelli, etc. $[49,50])$ in the early 2000s, based on a balance between dissipative and potential restoring forces. Slightly later a series of refinements were provided by Dal Maso's school [17], and also by other authors (such as, e.g., Ortiz, Francfort [18], De Simone, Neff [24]), some of them grounding their approach on the energy dissipation principle and De Giorgi's theory of minimizing movements. In the 80ies and 90ies, computational plasticity has been developed, based on strain incremental schemes, see for instance the classical textbooks of Hughes and Simo [64] and Han and Reddy [32].

6.2. Conventional (0th-order) elasto-plasticity models. Conventional models of small strain elasto-plasticity start with the following postulate. It is assumed that $\varepsilon^{e}=\mathbb{A}^{-1} \sigma$ is the elastic strain, with $\mathbb{A}$ the isotropic elasticity tensor and $\sigma$ the Cauchy stress tensor. Then, the eigenstrain is called plastic strain, $\bar{\varepsilon}=\varepsilon^{p}$, and there exists a vector field $u$ called the displacement field, satisfying

$$
e(u)=\varepsilon^{e}+\varepsilon^{p}
$$

Whereas at time $t$, the elastic strain $\varepsilon^{e}(t)$ obeys the elasticity system

$$
-\operatorname{div}\left(\mathbb{A} \varepsilon^{e}(t)\right)=f(t) \text { in } \Omega, \quad\left(\mathbb{A} \varepsilon^{e}(t)\right) N=g(t) \text { on } \Gamma_{N},
$$

the plastic strain satisfies other laws, called the flow rules. These are based on another series of postulates. Before recalling these rules, it is important to have in mind three facts regarding (6.2).

(i) The partition is local, i.e., $\varepsilon(x)=\varepsilon^{e}(x)+\varepsilon^{p}(x)$ for any $x \in \Omega$ and is purely of physical nature, that is, there is not any sort of mathematical structure behind it.

(ii) The geometric meaning of $\varepsilon$ as an intrinsic metric has been lost, since each part has its own definition, given by solving some equations, whereas the total deformation is defined by their sum. 
(iii) By (6.2), it is conventionally postulated that the total deformation is compatible, that is, that there exists a displacement field $u$ such that $\varepsilon=\nabla^{S} u=e(u)$. This statement is not justified by any mathematical argument and the adoption of this hypothesis is made for simplicity. Indeed it automatically implies that the incompatibilities of elastic and plastic parts mutually compensate, without the need to let the flow rules comply with this property.

As for the plastic part, following Moreau [52] it is assumed that:

- There exists a compact and convex subset $\mathcal{K}$ of symmetric $3 \times 3$-matrices such that the condition $\sigma \in \mathcal{K}$ is always satisfied. The yield surface is represented by the boundary $\partial \mathcal{K}$. Moreover, in the general context of plasticity with hardening, the elastic domain $\mathcal{K}(t)$ at time $t$ depends on $\sigma(t)$, to account for the back-stress tensor, and we write $\mathcal{K}(t)$ to mean $\mathcal{K}(\sigma(t))$.

- Let $I_{\mathcal{K}}$ be the indicator function of $\mathcal{K}$, i.e. $I_{\mathcal{K}}(\eta)=0$ if $\eta \in K$ and $I_{\mathcal{K}}(\eta)=+\infty$ if $\eta \notin \mathcal{K}$. Then the so-called associated flow rule (a special case commonly used) can be written as

$$
\left\{\begin{array}{ccc}
\sigma(t) \in \operatorname{int} \mathcal{K}(t) & \Rightarrow & \dot{\varepsilon}^{p}(t)=0 \\
\sigma(t) \in \partial \mathcal{K}(t) & \Rightarrow & \dot{\varepsilon}^{p}(t) \in \partial I_{\mathcal{K}(t)}(\sigma(t)) \quad \Leftrightarrow \quad(\eta-\sigma(t)) \cdot \dot{\varepsilon}^{p}(t) \leq 0, \forall \eta \in \mathcal{K}(t) .
\end{array}\right.
$$

Here $\partial I_{\mathcal{K}(t)}$ is the normal cone $N_{\mathcal{K}}(\sigma(t))$ and $\sigma(t) \in \operatorname{int} \mathcal{K}(t) \Leftrightarrow N_{\mathcal{K}}(\sigma(t))=\{0\}$. This formalism is to be compared with the model of Hill and Rice [34,57], which is summarized as follows:

- Introduce the dissipation potential $\mathcal{D}\left(\dot{\varepsilon}^{p}\right):=\sup \left\{\eta \cdot \dot{\varepsilon}^{p} \mid \eta \in \mathcal{K}(t)\right\}$, that is the support function of $\mathcal{K}(t)$. Convex calculus entails

$$
\mathcal{D}\left(\dot{\varepsilon}^{p}\right)=\sigma(t) \cdot \dot{\varepsilon}^{p} \Leftrightarrow \sigma(t) \in \partial \mathcal{D}\left(\dot{\varepsilon}^{p}\right) .
$$

- It is easily proven that the two formalisms are equivalent [32]:

$$
(6.4) \Leftrightarrow(6.5) \text {. }
$$

Note that the $\Leftarrow$ implication shows that Hill and Rice formalism does not require the notion of elastic domain and yield surface, which are obtained as consequences. However they postulate the existence of a potential $\mathcal{D}$ that is convex, positively homogeneous and lower semicontinuous. Thus we see that in both cases the model is strongly based on convex analysis.

\section{Gradient elasto-Plasticity for continua with Dislocations: TOWARDS AN INCOMPATIBILITY-DRIVEN MODEL}

7.1. The size effect. Zeroth-order models as described in Section 6 may not be sufficient with a view to several technological applications. For instance, it is essential for micro-electronics devices (such as silicon wafers for semiconductor production [53]) to accurately identify the mechanical properties of micro-structured materials, since there exist important strength differences that result from modification of the material micro-structural characteristics with changing size (where in general smaller sizes correlate with stronger responses). Indeed, the mechanical properties of micro-structured materials (e.g., yield strength, strain-hardening rate) with small-scale structures are extremely size-sensitive, and the increase in strength with decreasing scale can be related to increasing the strain gradients. For instance, industrial silicon is produced by the growth of a crystal seed, which by definition incorporates all material sizes and various types of microstructures (individual point-defects, voids, dislocations, volumic clusters, dislocations): present in the small-size material, they will grow together with the crystal and form defect structures of various sizes [53,74]. Further, serious issues prevent the use of classical (local) theories of plasticity and fracture (see [26] or [1]), since classical continuum mechanics cannot accurately capture size effects and highly localized deformations. On the other hand, atomistic simulations are out of reach in terms of computational cost, and therefore are restricted to small samples. In order to address the size effect problem, the so-called gradient plasticity models have gained an increasing interest in the scientific and technological communities. 
7.2. Gradient models. The success of gradient theories stems from the incorporation of a microstructural length scale parameter. Indeed, it is a general feature that gradient-theories do not assume stress as function of the sole history of strain at a point $x$, rather they take into account possible interactions with other material points in its neighbourhood. For example, the internal state variable that is responsible for isotropic hardening in classical plasticity theory is the effective plastic strain $p$, that, in a non-local media can be replaced by a weighted average $\bar{p}(x)=\int_{V} p(x+$ $\xi) h(\xi) d x$, where $x$ is the point of interest, $\xi$ is the size of the localized plastic zone, and $h(\xi)$ is a weighting function. Then $\bar{p}(x)$ can be approximated in terms of $p(x), L \nabla p(x)$ and $L^{2} \nabla^{2} p(x)$, for a certain characteristic length $L$. The physical basis of the gradient plasticity theory rests on theoretical developments of geometrically necessary dislocations (GNDs), see Daya Reddy, Gurtin and Neff works for instance $[8,21,30,31]$. For instance, the micro-mechanical modeling of the inelastic material behavior of metallic single crystals is based on the fact that resistance to glide is due to random trapping of mobile dislocations, "statistically stored" dislocations (SSDs), and acting as obstacles to further dislocation motion. On the other hand, the GND are related to the elastic strain incompatibility and are responsible for the observed macroscopic plastic behaviour, as stated by the famous Kröner's relation [41] "inc $\epsilon=$ Curl $\kappa$ " with $\kappa$ related to dislocation density. In the last two decades, another class of gradient theories was introduced, assuming higher-order gradients of the plastic strain field, as proposed in our model. These theories are a particular case of the generalized continua, such as continua with micro-structure, which were inspired by the pioneering work of the Cosserat brothers ${ }^{8}[16]$.

7.3. Our approach: a gradient model based on the strain incompatibility. We believe that the intrinsic point of view together with continuum-and gradient-based theories as proposed by our approach (see Sections 8 and 9) are needed to bridge the gap between classical continuum and micro-mechanical theories. The main feature of our model is that we focus on dislocation micro-structure in single crystals with a novel continuum model making an explicit link between plasticity and dislocations, and based on a novel model paradigm. As we will explain, the model we propose is of gradient type, involving the curl and the incompatibility of the strain. Further, the deformation tensor is seen as a metric in the aforementioned geometric sense. In particular we do not distinguish between elastic or plastic deformations.

The novel approach we propose has been introduced and discussed in [4]. In our model, neither of the three above postulates are considered. Our paradigm is radically different and our approach is based on the following rationales.

(1) Strain rate is prefered to strain and is given the following, primordial definition. Identify three fibers at $x$, denoted by $a_{1}, a_{2}, a_{3}$, which at time $t$ are oriented along the axes of a Cartesian coordinate system and of unit lengths. Then the deformation rate is defined at $x$ as (see, e.g., $[20,67])$

$$
d_{i j}(t)=\frac{1}{2}\left(\frac{d}{d t}\left(a_{i} \cdot a_{j}\right)\right)_{t} .
$$

Having fixed an initial time $t_{0}=0$, the time integral of the objective tensor $d$, called the strain or deformation tensor reads $\varepsilon(t)=\int_{0}^{t} d(s) d s$. Note that (7.1) holds for infinitesimal as well as for finite strains and hence one is not forced to specify the quantitative nature of the deformations before they take place.

(2) This strain defined in this fashion is neither elastic nor plastic, it simply has a compatible and an incompatible part, that are given by a structure theorem called Beltrami decomposition [46]:

$$
\varepsilon=\nabla^{S} u+E^{0} .
$$

As opposed to elastic-plastic splittings this decomposition is unique once boundary conditions for $u$ are prescribed. Moreover, while $\varepsilon$ is an objective field (in a general sense

\footnotetext{
${ }^{8}$ Indeed, the Cosserat (or micro-polar) continuum enhances the kinematic description of deformation by an additional field of local rotations.
} 
discussed in section 9 , neither $\nabla^{S} u$ nor $E^{0}$ are objective. Therefore the model will be constructed upon $\varepsilon$ and its derivatives.

(3) The governing equations should generalize classical linear elasticity in the sense that it must take into account the possible strain incompatibility. The idea behind is that the model should explicitely account for the physical cause of plasticity: the presence and motion of dislocations as microstructural perturbations.

As detailed below, the key point upon which our model relies is the fact that strain incompatibility is directly related to the density of dislocations by (4.3). Moreover, our model involves a new tangent material coefficient $\ell$, with the dimension of a force, representing the resistance of matter against incompatibility. In general, this scalar is space- and time-dependent, and evolves with the course of (at first quasi-static) deformation. If $\ell$ depends on space only, we proved in [2] that our model is a special case of the classical Mindlin gradient elasticity [51], whereas for a time-dependent $\ell$, it gives rise to a drastically new approach and a novel nonlinear plasticity model in direct relation with dislocation motion.

A difficult modelling problem that we have not yet achieved is to find an appropriate evolution equation for the incompatibility modulus $\ell$. In principle it should be derived based on dislocation mechanics as a function of temperature, strain, strain rate, and a set of measurable micro-structural physical parameters. Further, due to the existence of plastic strain gradient terms, higher-order boundary conditions are required on both external (free surfaces) and internal boundary (interfaces) regions where plastic deformation occurs. Also, these higher-order boundary conditions, which are motivated from the physical understanding of the dislocation mechanics, may vary with the course of plastic deformation. It is a further principle of our approach that boundary conditions should be naturally integrated in the functional framework of our equations. Hence internal transmission conditions are only byproducts of weak formulations.

7.4. Link with classical elasto-plasticity models. Recall that classical linearized elasto-plasticity models are based on the a priori decomposition $\varepsilon^{\text {tot }}=\varepsilon^{\mathrm{e}}+\varepsilon^{\mathrm{p}}$, where the total strain $\varepsilon^{\text {tot }}$ is compatible (inc $\varepsilon^{\text {tot }}=0$ ), the elastic strain $\varepsilon^{\mathrm{e}}$ is derived from the Cauchy stress by Hooke's law, and the plastic strain $\varepsilon^{\mathrm{p}}$ obeys flow rules. We now compare this decomposition with the Beltrami decomposition $\varepsilon=\nabla^{S} u+\varepsilon^{0}$. Since inc $\varepsilon^{\text {tot }}=$ inc $\nabla^{S} u=0$, there exists a vector field $w$ (see [46]) such that $\varepsilon^{\text {tot }}=\nabla^{S} u-\nabla^{S} w$ and we can write

$$
\varepsilon^{\text {tot }}=\nabla^{S} u-\nabla^{S} w=-\left(\varepsilon^{0}+\nabla^{S} w\right)+\left(\nabla^{S} u+\varepsilon^{0}\right) .
$$

We then recognize $\nabla^{S} u+\varepsilon^{0}$ as the strain $\varepsilon$. The correspondence with the Beltrami decomposition $\varepsilon=\nabla^{S} u+\varepsilon^{0}$ can be made upon setting $\varepsilon^{\mathrm{e}}=\varepsilon, \varepsilon^{\mathrm{p}}=-\left(\varepsilon^{0}+\nabla^{S} w\right)$.

The interpretation is the following (see Fig. 1): for us, $\varepsilon$ represents the deformation from a reference state, say state $A$ to a neighbour state $B$ of the same material. It can be viewed as the composition of the incompatible deformation $\varepsilon^{0}$ from state $A$ to an intermediate state $A^{\prime}$, and the compatible deformation $\nabla^{S} u$ from $A^{\prime}$ to $B$. In the classical approach, another configuration $A^{\prime \prime}$ serves as reference configuration. The total deformation $\varepsilon^{\text {tot }}$ from $A^{\prime \prime}$ to $B$ is the sum of the plastic deformation $\varepsilon^{\mathrm{p}}=-\left(\varepsilon^{0}+\nabla^{S} w\right)$ from $A^{\prime \prime}$ to $A$ and the elastic deformation $\varepsilon^{\mathrm{e}}=\varepsilon$ from $A$ to $B$. Therefore, plastic effects play somehow the role of configurational forces [29] that account for the change of reference configuration. Of course, choosing $w=0$ (thus $A^{\prime \prime}=A^{\prime}$ ) would be a choice of simplicity, but in that case $\varepsilon^{\mathrm{p}}$ would be identified with $-\varepsilon^{0}$, hence it would not be trace-free as assumed in some standard flow rules. We emphasize the arbirariness of A", whereas A' is uniquely determined from Beltrami decomposition. However, a significant difference between the kinematical frameworks of the two approaches is that $\varepsilon^{\mathrm{p}}$ is usually supposed trace-free whereas $\varepsilon^{0}$ is divergence-free. For us, incompressibility could be realized by an enrichment of the model, with possible additional variables and equations, but it is not prescribed a priori in the general setting. 


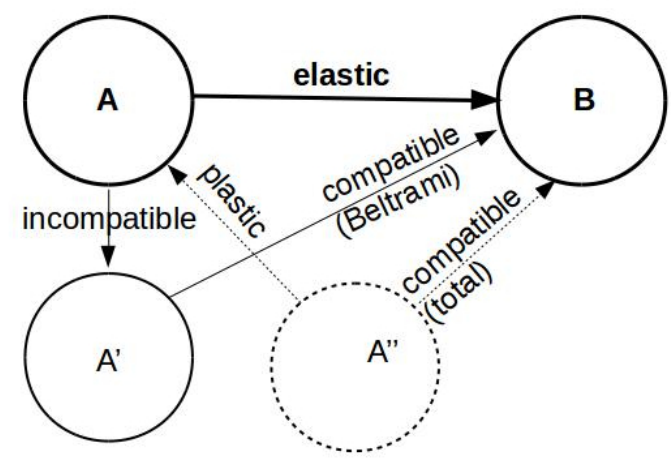

Figure 1. The Beltrami decomposition $\left(A \rightarrow A^{\prime} \rightarrow B\right)$ vs the standard elastic/plastic decomposition $\left(A^{\prime \prime} \rightarrow A \rightarrow B\right)$

\section{ThE INCOMPATIBILITY OPERATOR: FUNCTIONAL FRAMEWORK}

We have seen so far that the notion of incompatibility is a crucial ingredient in the modeling of dislocated crystals and therefore in the understanding of the plastic behavior of solids. From the mathematical point of view, inc is a second order differential operator that acts on (usually symmetric) tensor fields. We address in this section the analysis of this operator in the framework of Sobolev spaces. The Beltrami ${ }^{9}$ decomposition asserts that any symmetric tensor field can be split into a divergence-free part and an incompatibility-free part. It appears therefore natural to study the incompatibility operator in divergence-free spaces. As orthogonal complements, incompatibility-free spaces will also be discussed.

Let $\Omega$ be a regular $\left(\mathcal{C}^{\infty}\right)$ bounded domain of $\mathbb{R}^{3}$. We denote by $\partial \Omega$ its boundary and by $N$ its outward unit normal. Moreover $\mathbb{S}^{3}$ denotes the set of symmetric 3-matrices.

8.1. Divergence-free lifting, Green formula and applications. We begin with the divergencefree lifting of traces of symmetric tensor fields. Set

$$
\tilde{H}^{3 / 2}\left(\partial \Omega, \mathbb{S}^{3}\right)=\left\{E \in H^{3 / 2}\left(\partial \Omega, \mathbb{S}^{3}\right): \int_{\partial \Omega} E N d S(x)=0\right\} .
$$

Theorem 8.1 (Divergence-free lifting [3]). Let $\mathbb{E} \in \tilde{H}^{3 / 2}\left(\partial \Omega, \mathbb{S}^{3}\right)$ and $\mathbb{G} \in H^{1 / 2}\left(\partial \Omega, \mathbb{S}^{3}\right)$. There exists $E \in H^{2}\left(\Omega, \mathbb{S}^{3}\right)$ such that

$$
\begin{cases}E=\mathbb{E} & \text { on } \partial \Omega \\ \left(\partial_{N} E\right)_{T}=\mathbb{G}_{T} & \text { on } \partial \Omega \\ \operatorname{div} E=0 & \text { in } \Omega\end{cases}
$$

where the subscript $T$ stands for the tangential part. In addition, such a lifting can be obtained through a linear continuous operator

$$
\mathcal{L}_{\partial \Omega}:(\mathbb{E}, \mathbb{G}) \in \tilde{H}^{3 / 2}\left(\partial \Omega, \mathbb{S}^{3}\right) \times H^{1 / 2}\left(\partial \Omega, \mathbb{S}^{3}\right) \mapsto E \in H^{2}\left(\Omega, \mathbb{S}^{3}\right) .
$$

\footnotetext{
${ }^{9}$ Eugenio Beltrami (1835-1900) is an Italian physicist and mathematician known in particular for his works on elasticity-stating the equilibrium equations of a body in terms of the stress in place of the strain [9]- but also in non-Euclidean geometries in the wake of Gauss and Riemann. He was indeed a friend of Riemann whom he met at Pisa university where he had a chair. Moreover, his chair of mathematical physics in Rome was later transmitted to Volterra in 1900. Vito Volterra (1860-1940) is presumably the first who gave a correct definition of dislocations and disclinations in [77]. It is thus not mere coincidence that the name of Beltrami will take a crucial place in our survey on incompatibility and dislocations. Neither that the second author of this survey started his study of dislocations with a book found in the main Scuola Normale library in Pisa in 2000 [38].
} 
Define the subset of $\mathcal{C}^{\infty}\left(\partial \Omega, \mathbb{S}^{3}\right)$

$$
\mathcal{G}=\left\{V \odot N, V \in \mathbb{R}^{3}\right\},
$$

with the notation $U \odot V:=(U \otimes V+V \otimes U) / 2$.

Lemma 8.2 (Dual trace space $[3])$. Every $\mathbb{E} \in H^{-3 / 2}\left(\partial \Omega, \mathbb{S}^{3}\right) / \mathcal{G}$ admits a unique representative $\tilde{\mathbb{E}}$ such that

$$
\int_{\partial \Omega} \tilde{\mathbb{E}} N d S(x)=0
$$

Moreover, the dual space of $\tilde{H}^{3 / 2}\left(\partial \Omega, \mathbb{S}^{3}\right)$ is canonically identified with $H^{-3 / 2}\left(\partial \Omega, \mathbb{S}^{3}\right) / \mathcal{G}$.

We define the spaces of symmetric tensor fields

$$
\begin{aligned}
H^{\operatorname{div}}\left(\Omega, \mathbb{S}^{3}\right) & :=\left\{E \in L^{2}\left(\Omega, \mathbb{S}^{3}\right): \operatorname{div} E \in L^{2}\left(\Omega, \mathbb{R}^{3}\right)\right\}, \\
H^{\text {inc }}\left(\Omega, \mathbb{S}^{3}\right) & :=\left\{E \in L^{2}\left(\Omega, \mathbb{S}^{3}\right): \operatorname{inc} E \in L^{2}\left(\Omega, \mathbb{S}^{3}\right)\right\},
\end{aligned}
$$

endowed with the norms defined by $\|E\|_{H^{\text {div }}}^{2}=\|E\|_{L^{2}}^{2}+\|\operatorname{div} E\|_{L^{2}}^{2},\|E\|_{H^{\text {inc }}}^{2}=\|E\|_{L^{2}}^{2}+\|$ inc $E \|_{L^{2}}^{2}$, respectively.

Recall that the Green formula for the divergence allows to define, for any $T \in H^{\operatorname{div}}\left(\Omega, \mathbb{S}^{3}\right)$, its normal trace $T N \in H^{-1 / 2}\left(\partial \Omega, \mathbb{R}^{3}\right)$ by

$$
\int_{\partial \Omega}(T N) \cdot \varphi d S(x):=\int_{\Omega}\left(\operatorname{div} T \cdot \tilde{\varphi}+T \cdot \nabla^{S} \tilde{\varphi}\right) d x \quad \forall \varphi \in H^{1 / 2}\left(\partial \Omega, \mathbb{R}^{3}\right),
$$

with $\tilde{\varphi} \in H^{1}\left(\Omega, \mathbb{R}^{3}\right)$ an arbitrary lifting of $\varphi$. For the incompatibility operator one has the following counterpart.

Lemma 8.3 (Green formula for the incompatibility [3]). Suppose that $T \in \mathcal{C}^{2}\left(\bar{\Omega}, \mathbb{S}^{3}\right)$ and $\eta \in$ $H^{2}\left(\Omega, \mathbb{S}^{3}\right)$. Then

$$
\int_{\Omega} T \cdot \operatorname{inc} \eta d x=\int_{\Omega} \operatorname{inc} T \cdot \eta d x+\int_{\partial \Omega} \mathcal{T}_{1}(T) \cdot \eta d S(x)+\int_{\partial \Omega} \mathcal{T}_{0}(T) \cdot \partial_{N} \eta d S(x)
$$

with the trace operators defined as

$$
\begin{aligned}
& \mathcal{T}_{0}(T):=(T \times N)^{T} \times N, \\
& \mathcal{T}_{1}(T):=\left(\operatorname{Curl}(T \times N)^{T}\right)^{S}+\left(\left(\partial_{N}+k\right) T \times N\right)^{t} \times N+\left(\operatorname{Curl}^{T} T \times N\right)^{S},
\end{aligned}
$$

where $k$ is twice the mean curvature of $\partial \Omega$ and $T^{S}=\left(T+T^{T}\right) / 2$. In addition, it holds

$$
\int_{\partial \Omega} \mathcal{T}_{1}(T) N d S(x)=0
$$

Note that $(T \times N)^{T} \times N$ is made of permutations of the tangential components of $T$. Alternative expressions of $\mathcal{T}_{1}(T)$ are derived in [3]. Therefore, we can define the traces $\mathcal{T}_{0}(T) \in H^{-1 / 2}\left(\partial \Omega, \mathbb{S}^{3}\right)$ and $\mathcal{T}_{1}(T) \in H^{-3 / 2}\left(\partial \Omega, \mathbb{S}^{3}\right) / \mathcal{G}$ for every $T \in H^{\text {inc }}\left(\Omega, \mathbb{S}^{3}\right)$ by

$$
\begin{aligned}
& \left\langle\mathcal{T}_{0}(T), \varphi_{0}\right\rangle=\int_{\Omega} T \cdot \operatorname{inc} \eta_{0} d x-\int_{\Omega} \text { inc } T \cdot \eta_{0} d x, \quad \forall \varphi_{0} \in H^{1 / 2}\left(\partial \Omega, \mathbb{S}^{3}\right), \\
& \left\langle\mathcal{T}_{1}(T), \varphi_{1}\right\rangle=\int_{\Omega} T \cdot \operatorname{inc} \eta_{1} d x-\int_{\Omega} \operatorname{inc} T \cdot \eta_{1} d x, \quad \forall \varphi_{1} \in \tilde{H}^{3 / 2}\left(\partial \Omega, \mathbb{S}^{3}\right),
\end{aligned}
$$

with $\eta_{0}=\mathcal{L}_{\partial \Omega}\left(0, \varphi_{0}\right)$ and $\eta_{1}=\mathcal{L}_{\partial \Omega}\left(\varphi_{1}, 0\right)$ (recall that $\mathcal{L}_{\partial \Omega}$ is the lifting operator defined in Theorem 8.1). In addition, by Lemma 8.2, $\mathcal{T}_{1}(T)$ admits a unique representative satisfying (8.5). By linearity of $\mathcal{L}_{\partial \Omega}$, this extends formula (8.2) to any functions $T \in H^{\text {inc }}\left(\Omega, \mathbb{S}^{3}\right)$ and $\eta \in H^{2}\left(\Omega, \mathbb{S}^{3}\right)$.

From the two Green formulas recalled above one immediately infers that:

- inc $\nabla^{S} v=0$ in the sense of distributions for all $v \in H^{1}\left(\Omega, \mathbb{R}^{3}\right)$;

- $\operatorname{div}$ inc $E=0$ in the sense of distributions for all $E \in H^{\mathrm{inc}}\left(\Omega, \mathbb{S}^{3}\right)$. 
In particular, if $E \in H^{\text {inc }}\left(\Omega, \mathbb{S}^{3}\right)$, then inc $E N$ is defined in $H^{-1 / 2}\left(\partial \Omega, \mathbb{R}^{3}\right)$ by

$$
\int_{\partial \Omega} \operatorname{inc} E N \cdot \varphi d x=\int_{\Omega} \operatorname{inc} E \cdot \nabla^{S} \varphi d x \quad \forall \varphi \in H^{1}\left(\Omega, \mathbb{R}^{3}\right) .
$$

Let $\Gamma$ be a smooth subset of $\partial \Omega$ and set

$$
H_{0}^{\text {inc }}\left(\Omega, \mathbb{S}^{3}\right)=\text { the closure of } \mathcal{D}\left(\Omega, \mathbb{S}^{3}\right) \text { in } H^{\text {inc }}\left(\Omega, \mathbb{S}^{3}\right) .
$$

Further properties of the trace operators are given below.

Proposition 8.4 (Trace properties [2]). $\quad$ (1) Let $v \in H^{1}\left(\Omega, \mathbb{R}^{3}\right)$ be such that $v=r$ on $\Gamma$ in the sense of traces, with $r$ a rigid displacement field. Then $\mathcal{T}_{0}\left(\nabla^{S} v\right)=\mathcal{T}_{1}\left(\nabla^{S} v\right)=0$ on $\Gamma$.

(2) We have the characterization

$$
H_{0}^{\text {inc }}\left(\Omega, \mathbb{S}^{3}\right)=\left\{E \in H^{\text {inc }}\left(\Omega, \mathbb{S}^{3}\right): \mathcal{T}_{0}(E)=\mathcal{T}_{1}(E)=0 \text { on } \partial \Omega\right\} .
$$

(3) If $E \in H_{0}^{\mathrm{inc}}\left(\Omega, \mathbb{S}^{3}\right)$ then inc $E N=0$ on $\partial \Omega$.

8.2. Saint-Venant compatibility conditions and Beltrami decomposition. We state the following two results in the setting of $L^{p}$ spaces for generality, although we will be concerned with $p=2$ only.

Theorem 8.5 (Saint-Venant compatibility conditions [46]). Assume that $\Omega$ is simply-connected. Let $p \in(1,+\infty)$ be a real number and let $E \in L^{p}\left(\Omega, \mathbb{S}^{3}\right)$. Then,

$$
\text { inc } E=0 \text { in } W^{-2, p}\left(\Omega, \mathbb{S}^{3}\right) \Longleftrightarrow E=\nabla^{S} v
$$

for some $v \in W^{1, p}\left(\Omega, \mathbb{R}^{3}\right)$. Moreover, $u$ is unique up to rigid displacements.

Theorem 8.6 (Beltrami decomposition [46]). Assume that $\Omega$ is simply-connected. Let $p \in(1,+\infty)$ be a real number and let $E \in L^{p}\left(\Omega, \mathbb{S}^{3}\right)$. Then, for any $v_{0} \in W^{1 / p, p}(\partial \Omega)$, there exists a unique $v \in W^{1, p}\left(\Omega, \mathbb{R}^{3}\right)$ with $v=v_{0}$ on $\partial \Omega$ and a unique $F \in L^{p}\left(\Omega, \mathbb{S}^{3}\right)$ with Curl $F \in L^{p}\left(\Omega, \mathbb{R}^{3 \times 3}\right)$, inc $F \in L^{p}\left(\Omega, \mathbb{S}^{3}\right)$, div $F=0$ and $F N=0$ on $\partial \Omega$ such that

$$
E=\nabla^{S} v+\text { inc } F \text {. }
$$

A variant of Saint-Venant's compatibility conditions in the presence of boundary conditions is the following.

Proposition 8.7 (Saint-Venant with boundary condition [2]). Assume that $\Omega$ is simply-connected. If $E \in L^{2}\left(\Omega, \mathbb{S}^{3}\right)$ satisfies

$$
\left\{\begin{array}{l}
\text { inc } E=0 \text { in } \Omega, \\
\mathcal{T}_{0}(E)=\mathcal{T}_{1}(E)=0 \text { on } \partial \Omega
\end{array}\right.
$$

then there exists $v \in H_{0}^{1}\left(\Omega, \mathbb{R}^{3}\right)$ such that $\nabla^{S} v=E$. Moreover, the map $E \in L^{2}\left(\Omega, \mathbb{S}^{3}\right) \mapsto v \in$ $H_{0}^{1}\left(\Omega, \mathbb{R}^{3}\right)$ is linear and continuous.

We assume from now on that $\Omega$ is simply-connected.

8.3. Orthogonal decompositions. For $\Gamma$ being a smooth subset of $\partial \Omega$, we define the sets

$$
\begin{aligned}
\mathcal{V} & =\left\{E \in L^{2}\left(\Omega, \mathbb{S}^{3}\right): \text { inc } E=0\right\}, \\
\mathcal{V}_{\Gamma}^{0} & =\left\{E \in \mathcal{V}: \mathcal{T}_{0}(E)=\mathcal{T}_{1}(E)=0 \text { on } \Gamma\right\}, \\
\mathcal{V}_{\Gamma}^{00} & =\left\{\nabla^{S} v: v \in H^{1}(\Omega), v=0 \text { on } \Gamma\right\}, \\
\mathcal{W} & =\left\{E \in L^{2}\left(\Omega, \mathbb{S}^{3}\right): \operatorname{div} E=0\right\}, \\
\mathcal{W}_{\Gamma}^{0} & =\{E \in \mathcal{W}: E N=0 \text { on } \Gamma\} .
\end{aligned}
$$

From what precedes we infer the following relations [2]:

$$
\mathcal{V}=\mathcal{V}_{\emptyset}^{0}=\mathcal{V}_{\emptyset}^{00} \quad \mathcal{V}_{\Gamma}^{00} \subset \mathcal{V}_{\Gamma}^{0}, \quad \mathcal{V}_{\partial \Omega}^{00}=\mathcal{V}_{\partial \Omega}^{0} .
$$

A refinement of the Beltrami decomposition is obtained as follows. 
Theorem 8.8 (Orthogonal decomposition of $L^{2}\left(\Omega, \mathbb{S}^{3}\right)[2]$ ). Assume that $\partial \Omega$ admits the partition $\partial \Omega=\Gamma_{1} \cup \Gamma_{2}$ with $\Gamma_{1} \cap \Gamma_{2}=\emptyset$. We have the orthogonal decomposition

$$
L^{2}\left(\Omega, \mathbb{S}^{3}\right)=\mathcal{V}_{\Gamma_{1}}^{00} \oplus \mathcal{W}_{\Gamma_{2}}^{0}
$$

Related to this decomposition, the following lemma will be useful.

Lemma 8.9 (Boundary orthogonality relation $[2])$. If $\mathbb{K} \in \mathcal{V}_{\Gamma_{1}}^{00}$ and inc $\hat{F} \in \mathcal{W}_{\Gamma_{2}}^{0}$ then

$$
\int_{\Gamma_{2}}\left(\mathcal{T}_{1}(\mathbb{K}) \cdot \hat{F}+\mathcal{T}_{0}(\mathbb{K}) \cdot \partial_{N} \hat{F}\right) d S(x)=0
$$

We now define the spaces with further differentiability properties

$$
\begin{aligned}
\mathcal{Z} & =\left\{E \in H^{\text {inc }}\left(\Omega, \mathbb{S}^{3}\right): \operatorname{div} E=0 \text { in } \Omega, E N=0 \text { on } \partial \Omega\right\}, \\
\mathcal{Z}_{0} & =\{E \in \mathcal{Z}: \operatorname{inc} E N=0 \text { on } \partial \Omega\}, \\
\mathcal{F} & =\left\{E \in H^{\text {inc }}\left(\Omega, \mathbb{S}^{3}\right): \text { inc } E N=0 \text { on } \partial \Omega\right\} .
\end{aligned}
$$

A straightforward consequence of Theorem 8.8 is the following.

Proposition 8.10. We have the orthogonal decompositions

$$
H^{\text {inc }}\left(\Omega, \mathbb{S}^{3}\right)=\mathcal{Z} \oplus \mathcal{V}, \quad \mathcal{F}=\mathcal{Z}_{0} \oplus \mathcal{V} .
$$

8.4. Boundary value problems for the incompatibility. If $E \in H^{\text {inc }}\left(\Omega, \mathbb{S}^{3}\right)$ is split into $E=E_{i}+E_{c}, E_{i} \in \mathcal{Z}, E_{c} \in \mathcal{V}$, then inc $E=$ inc $E_{i}$. Thus, a Poincaré inequality for the incompatibility is naturally sought in $\mathcal{Z}$ or one of its subspaces. In fact, the following holds.

Proposition 8.11 (First Poincaré inequality [2]). There exists $C>0$ such that, for all $E \in \mathcal{Z}$,

$$
\|E\|_{H^{1}} \leq C \| \text { inc } E \|_{L^{2}} .
$$

As a straightforward consequence, given $\mathbb{K} \in L^{2}\left(\Omega, \mathbb{S}^{3}\right)$ and $\mathbb{B}$ a symmetric uniformly positive definite fourth order tensor field, we infer by the Lax-Milgram theorem the existence of a unique $E \in \mathcal{Z}$ such that

$$
\int_{\Omega} \mathbb{B} \text { inc } E \cdot \operatorname{inc} \hat{E} d x=\int_{\Omega} \mathbb{K} \cdot \hat{E} d x \quad \forall \hat{E} \in \mathcal{Z}
$$

The same result holds if $\mathcal{Z}_{0}$ is substituted for $\mathcal{Z}$. If $\operatorname{div} \mathbb{K}=0$ in $\Omega, \mathbb{K} N=0$ on $\partial \Omega$ and $E \in \mathcal{Z}$ solves (8.8), then Proposition 8.10 shows that (8.8) holds actually for any $\hat{E}$ in $H^{\text {inc }}\left(\Omega, \mathbb{S}^{3}\right)$. This yields the strong form

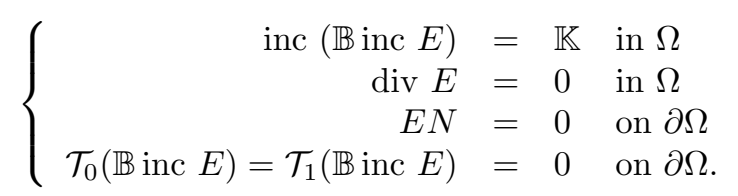

Dirichlet-type boundary conditions can be considered through the space

$$
\mathcal{H}_{0}=\left\{E \in H^{2}\left(\Omega, \mathbb{S}^{3}\right): \operatorname{div} E=0 \text { in } \Omega, E=\left(\partial_{N} E \times N\right)^{T} \times N=0 \text { on } \partial \Omega\right\} .
$$

Observe from the Green formula that the boundary conditions in $\mathcal{H}_{0}$ are dual to the trace operators $\mathcal{T}_{1}$ and $\mathcal{T}_{0}$. We have the following Poincaré inequality:

Proposition 8.12 (Second Poincaré inequality [3]). There exists $C>0$ such that, for all $E \in \mathcal{H}_{0}$,

$$
\|E\|_{H^{2}} \leq C \| \text { inc } E \|_{L^{2}} .
$$

Obviously, given $\mathbb{K} \in L^{2}\left(\Omega, \mathbb{S}^{3}\right)$ and $\mathbb{B}$ a symmetric uniformly positive definite fourth order tensor field, there is a unique $E \in \mathcal{H}_{0}$ such that

$$
\int_{\Omega} \mathbb{B} \text { inc } E \cdot \text { inc } \hat{E} d x=\int_{\Omega} \mathbb{K} \cdot \hat{E} d x \quad \forall \hat{E} \in \mathcal{H}_{0} \text {. }
$$


Nonhomogeneous boundary conditions can also be prescribed, using Theorem 8.1. In order to identify the strong form of (8.9), we first note that, if $E \in \mathcal{H}_{0}$ solves (8.9), then there exists a Lagrange multiplier (see e.g. [11]) $p \in L^{2}\left(\Omega, \mathbb{R}^{3}\right)$ such that

$$
\int_{\Omega} \mathbb{B} \operatorname{inc} E \cdot \operatorname{inc} \hat{E} d x-\int_{\Omega} p \cdot \operatorname{div} \hat{E} d x=\int_{\Omega} \mathbb{K} \cdot \hat{E} d x
$$

for all $\hat{E} \in H^{2}\left(\Omega, \mathbb{S}^{3}\right)$ with $\hat{E}=\left(\partial_{N} \hat{E} \times N\right)^{T} \times N=0$ on $\partial \Omega$. Therefore the strong form reads

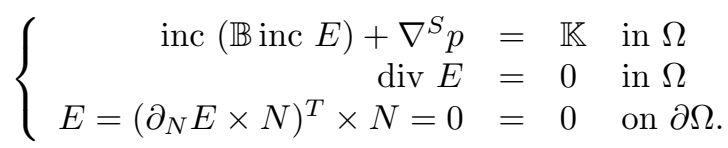

\section{TOWARDS AN INTRINSIC APPROACH TO LINEARIZED ELASTO-PLASTICITY}

9.1. Objectivity and principle of virtual powers. Consider a macroscopic solid represented by the domain $\Omega$ and subject to external loading. We place ourselves in a linearized setting, that is, our aim is to descibe the deformation of the solid between two close configurations (see Fig. 1), when the load admits a small increment. The evolution of this tangent modeling between increments will be discussed later, but an integrated nonlinear approach is currently out of our scope.

We will use the principle of virtual powers to describe the internal efforts acting within the solid and derive balance equations. In this framework, efforts are represented by powers, rather than forces. Two types of efforts are treated separately: the external efforts (the exterior medium acts on the body) and the internal efforts (the matter acts on itself). The corresponding powers are linear functionals that act on kinematical descriptors also called test or virtual fields. This is why we speak of virtual powers. The choice of these kinematical descriptors is of paramount importance.

A kinematical field is said objective if it is independent of the observer. The classical mathematical definition is the following: it is a scalar, vector, or tensor field that obeys the standard rules of transformation for such quantities through a roto-translation of the frame with arbitrary speed. In this setting, it is well-known that the velocity is not objective, whereas its symmetric gradient is. Nonetheless, we have seen that in the presence of defects the notion of velocity is not always well-defined. More general kinematical concepts are the geometric data of the solid seen as a Riemannian manifold. For us, only these fields (or their time rates) will be considered as objective. Hence, objective kinematical descriptors will be built upon the metric $g$. Typically, we will consider the strain $E:=g-\mathbb{I}$ and its rate $\dot{E}$.

In our model we assume that the internal virtual power is a continuous linear functional on $L^{2}\left(\Omega, \mathbb{S}^{3}\right)$, the set of virtual strain rates. By Riesz representation, there exists a generalized force field $\Sigma \in L^{2}\left(\Omega, \mathbb{S}^{3}\right)$ such that

$$
\mathcal{P}_{(i)}(\hat{E})=\int_{\Omega} \Sigma \cdot \hat{E} d x \quad \forall \hat{E} \in L^{2}\left(\Omega, \mathbb{S}^{3}\right) .
$$

Unlike its internal counterpart, the virtual external power is a linear functional against kinematical fields which may be non-objective (see, e.g., [47]). Typically, the velocity is considered in order to represent standard forces. However, in our framework, the velocity is only a byproduct of the strain rate, defined by orthogonal projection onto an appropriate function space. Therefore, it is natural to assume that the virtual external power is a linear functional on the set of virtual strain rates and we write

$$
\mathcal{P}_{(e)}(\hat{E})=\int_{\Omega} \mathbb{K} \cdot \hat{E} d x \quad \forall \hat{E} \in L^{2}\left(\Omega, \mathbb{S}^{3}\right),
$$

for some $\mathbb{K} \in L^{2}\left(\Omega, \mathbb{S}^{3}\right)$. The interpretation of $\mathbb{K}$ will be discussed in section 9.4. In the absence of inertial effects, the principle of virtual powers reads

$$
\mathcal{P}_{(i)}(\hat{E})=\mathcal{P}_{(e)}(\hat{E}),
$$


for all $\hat{E}$ satisfying possible kinematical constraints.

9.2. Constitutive law. We assume that the generalized force $\Sigma$ is a function of the local geometric data of the solid. This relation is called constitutive law. In our linearized framework, we assume that $\Sigma(x)$ is expressed as a linear function of the pair composed of $E(x)$, the strain at point $x$, and inc $E(x)$, the linearized Riemannian curvature. Therefore we can write

$$
\Sigma(x)=\mathbb{A} E(x)+\mathbb{B} \text { inc } E(x),
$$

for some fourth-order tensors $\mathbb{A}$ and $\mathbb{B}$.

In classical elasticity one has inc $E=0$, so that $\mathbb{A}$ is recognized as the Hooke tensor of the material. We place ourselves in the isotropic case where the standard expression

$$
\mathbb{A}=\lambda \mathbb{I}_{2} \otimes \mathbb{I}_{2}+2 \mu \mathbb{I}_{4}
$$

holds, with $(\lambda, \mu)$ the Lamé coefficients.

Tensor $\mathbb{B}$ is a new object. An assumption of consistency with linear elasticity will reduce its expression. First, let us emphasize that classical compatible elasticity formally corresponds to $\mathbb{B}$ 'large': indeed, the term $\mathbb{B}$ inc $E$ penalizes incompatible deformations. Second, the equations of linear elasticity are derived from the principle of virtual powers considering compatible test fields $\hat{E}=\nabla^{S} \hat{v}$. Thus, we want that following property be fulfilled: if $\mathbb{B}$ is homogeneous, then

$$
\int_{\Omega} \mathbb{B} \text { inc } E \cdot \hat{E} d x=0 \quad \forall \hat{E}=\nabla^{S} \hat{v}, \hat{v} \in \mathcal{D}\left(\Omega, \mathbb{R}^{3}\right) .
$$

From the Green formula, it turns out that $\mathbb{B}=\ell \mathbb{I}_{4}$, where $\ell$ is a scalar coefficient which we call incompatibility modulus, is a sufficient condition. Eventually, we arrive at the constitutive law

$$
\Sigma=\mathbb{A} E+\ell \text { inc } E .
$$

Different derivations of (9.4), based on Mindlin's theory of gradient elasticity [51], are given in $[4,44]$.

9.3. Equilibrium equations. Plugging (9.1), (9.2) and (9.4) into (9.3) entails that

$$
\int_{\Omega}(\mathbb{A} E+\ell \operatorname{inc} E) \cdot \hat{E} d x=\int_{\Omega} \mathbb{K} \cdot \hat{E} d x,
$$

for all admissible $\hat{E}$. In the following, we will not consider any kinematical constraint, whereby (9.5) reduces to

$$
\mathbb{A} E+\ell \text { inc } E=\mathbb{K}
$$

9.4. Interpretation of the external power and kinematical framework. Assume that $\partial \Omega=$ $\Gamma_{1} \cup \Gamma_{2}, \Gamma_{1} \cap \Gamma_{2}=\emptyset$, and take $\hat{E} \in L^{2}\left(\Omega, \mathbb{S}^{3}\right)$. In view of Theorem 8.8, we have the unique decomposition $\hat{E}=\nabla^{S} \hat{v}+\operatorname{inc} \hat{F}$ with $\nabla^{S} \hat{v} \in \mathcal{V}_{\Gamma_{1}}^{00}$ and inc $\hat{F} \in \mathcal{W}_{\Gamma_{2}}^{0}$. The Green formula yields

$$
\begin{aligned}
\mathcal{P}_{(e)}(\hat{E})=\int_{\Omega} \mathbb{K} \cdot \hat{E} d x= & -\int_{\Omega} \operatorname{div} \mathbb{K} \cdot \hat{v} d x+\int_{\Gamma_{2}} \mathbb{K} N \cdot \hat{v} d S(x)+\int_{\Omega} \operatorname{inc} \mathbb{K} \cdot \hat{F} d x \\
& +\int_{\partial \Omega}\left(\mathcal{T}_{1}(\mathbb{K}) \cdot \hat{F}+\mathcal{T}_{0}(\mathbb{K}) \cdot \partial_{N} \hat{F}\right) d S(x) .
\end{aligned}
$$

Therefore, $f:=-\operatorname{div} \mathbb{K}$ is identified with a body force, and $g:=\mathbb{K} N$ is identified with a surface load on $\Gamma_{2}$. Now, if $\mathbb{K} \in \mathcal{V}_{\Gamma_{1}}^{00}$ the last two integrals of (9.7) vanish by virtue of Lemma 8.9. Then (9.7) rewrites as the classical expression of the external power in linear elasticity

$$
\int_{\Omega} \mathbb{K} \cdot \hat{E} d x=\int_{\Omega} f \cdot \hat{v} d x+\int_{\Gamma_{2}} g \cdot \hat{v} d S(x) .
$$


To sum up, given $f \in L^{2}\left(\Omega, \mathbb{R}^{3}\right)$ and $g \in H^{-1 / 2}\left(\Gamma_{2}, \mathbb{R}^{3}\right)$, the standard external power is obtained after solving

$$
\left\{\begin{array}{l}
-\operatorname{div} \nabla^{S} w=f \text { in } \Omega \\
w=0 \text { on } \Gamma_{1} \\
\nabla^{S} w N=g \text { on } \Gamma_{2}
\end{array}\right.
$$

and setting $\mathbb{K}=\nabla^{S} w \in L^{2}\left(\Omega, \mathbb{S}^{3}\right)$.

9.5. Existence results and elastic limit. The main result of this section is the following.

Theorem 9.1 (Well-posedness [2]). Assume $\Omega$ is simply connected. Let $\mathbb{K} \in L^{2}\left(\Omega, \mathbb{S}^{3}\right)$. Let $C$ be the Poincaré constant of Proposition 8.11. If $\mathbb{A}$ is uniformly positive definite and $|\ell|>C|\mathbb{A}|$ a.e., then there exists one and only one $E \in \mathcal{F}$ such that

$$
\mathbb{A} E+\ell \text { inc } E=\mathbb{K} \text {. }
$$

Moreover we have the a priori estimate

$$
\| \text { inc } E\left\|_{L^{2}} \leq \frac{\left\|\ell^{-1} \mathbb{A}\right\|_{L^{\infty}}}{1-C\left\|\ell^{-1} \mathbb{A}\right\|_{L^{\infty}}}\right\| \mathbb{A}^{-1} \mathbb{K} \|_{L^{2}} .
$$

The essential boundary condition inc $E N=0$ (no incompatibility flux) appears naturally in the proof, through integrations by parts. It models the fact that the outside of $\Omega$ has a purely elastic behavior, however future work should go further into this point, see the discussion in [2] where an alternative condition for the modeling of free boundaries is also analyzed. Inequality (9.11) shows that inc $E$ tends to 0 as $|\ell| \rightarrow+\infty$. The following result is more precise.

Theorem 9.2 (Elastic limit [2]). Assume that $\mathbb{A}, \mathbb{K}$ are fixed, $\ell$ is constant, $E^{\ell} \in \mathcal{F}, \mathbb{A} E^{\ell}+$ $\ell$ inc $E^{\ell}=\mathbb{K}$ in $\Omega$. There exists a unique $E^{\infty} \in \mathcal{V}$ such that

$$
\int_{\Omega} \mathbb{A} E^{\infty} \cdot \hat{E} d x=\int_{\Omega} \mathbb{K} \cdot \hat{E} d x \quad \forall \hat{E} \in \mathcal{V} .
$$

Moreover $\left\|E^{\ell}-E^{\infty}\right\|_{L^{2}} \rightarrow 0$ when $|\ell| \rightarrow+\infty$.

Theorem 9.2 shows that the standard linear elasticity problem with Neumann boundary condition is retrieved as a limit case when $|\ell| \rightarrow+\infty$. The modeling of Dirichlet type boundary conditions require further work.

From our derivations so far, the question of the sign of $\ell$ has not been fixed. The example of the bar in traction shown below suggests that $\ell$ be negative to obtain realistic solutions.

9.6. Example: bar in traction. We present a variant of an example treated in [2]. Consider the domain $\Omega=\mathbb{R}^{2} \times(-h, h)$, for a given $h>0$. Although the existence theory has been carried out for bounded domains, the semi-infinite case will allow analytical calculations through ordinary differential equations. We assume a uniform vertical traction on the planes $\{z= \pm h\}$. In view of (9.7)-(9.9) we obtain

$$
\mathbb{K}=\left(\begin{array}{lll}
0 & 0 & 0 \\
0 & 0 & 0 \\
0 & 0 & 1
\end{array}\right)
$$

We search for a strain field of form

$$
E=\left(\begin{array}{ccc}
\varphi & 0 & 0 \\
0 & \varphi & 0 \\
0 & 0 & \psi
\end{array}\right)
$$

where $\varphi, \psi$ are functions of the $z$ variable. One has

$$
\mathbb{A} E=\left(\begin{array}{ccc}
2(\lambda+\mu) \varphi+\lambda \psi & 0 & 0 \\
0 & 2(\lambda+\mu) \varphi+\lambda \psi & 0 \\
0 & 0 & 2 \lambda \varphi+(\lambda+2 \mu) \psi
\end{array}\right) \text {, inc } E=\left(\begin{array}{ccc}
\varphi^{\prime \prime} & 0 & 0 \\
0 & \varphi^{\prime \prime} & 0 \\
0 & 0 & 0
\end{array}\right) \text {, }
$$



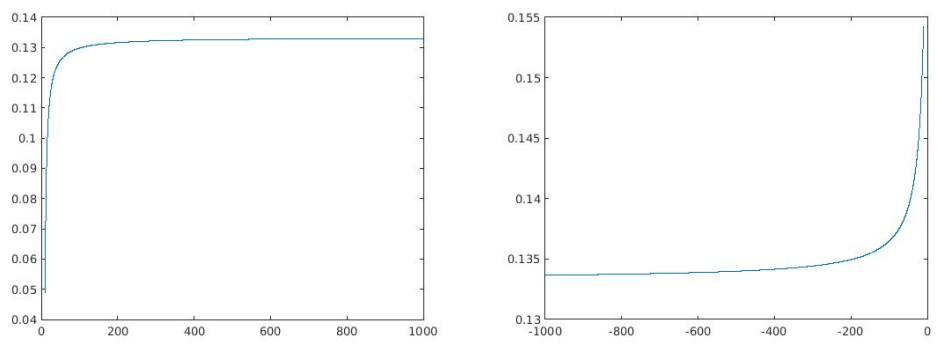

FiguRE 2. External work as a function of $\ell$ for $\ell>0$ (left) and $\ell<0$ (right)

whereby $\mathbb{A} E+\ell$ inc $E=\mathbb{K}$ if and only if

$$
\left\{\begin{array}{l}
2(\lambda+\mu) \varphi+\lambda \psi+\ell \varphi^{\prime \prime}=0 \\
2 \lambda \varphi+(\lambda+2 \mu) \psi=1
\end{array}\right.
$$

Substitution leads to

$$
\begin{gathered}
\psi=\frac{1}{\lambda+2 \mu}(1-2 \lambda \varphi), \\
2 \mu(3 \lambda+2 \mu) \varphi+\ell(\lambda+2 \mu) \varphi^{\prime \prime}=-\lambda .
\end{gathered}
$$

Due to the unboundedness of $\Omega$ the above equation has no unique solution. Therefore we prescribe $\varphi( \pm h)=0$. This entails

$$
\varphi(z)=\left\{\begin{array}{ll}
\frac{-\lambda}{2 \mu(3 \lambda+2 \mu)}\left(1-\frac{\cos \frac{\omega z}{\sqrt{\ell}}}{\cos \frac{\omega h}{\sqrt{\ell}}}\right) & \text { if } \ell>0,
\end{array} \quad \text { with } \omega=\sqrt{\frac{2 \mu(3 \lambda+2 \mu)}{\lambda+2 \mu} .}\right.
$$

The external work is obtained as

$$
W=\int_{-h}^{h} \psi(z) d z
$$

with $\psi$ given by (9.14), i.e.,

$$
W= \begin{cases}\frac{2 h}{\lambda+2 \mu}\left[1+\frac{\lambda^{2}}{\mu(3 \lambda+2 \mu)}\left(1-\frac{\sqrt{\ell}}{\omega h} \tan \frac{\omega h}{\sqrt{\ell}}\right)\right] & \text { if } \ell>0 \\ \frac{2 h}{\lambda+2 \mu}\left[1+\frac{\lambda^{2}}{\mu(3 \lambda+2 \mu)}\left(1-\frac{\sqrt{|\ell|}}{\omega h} \tanh \frac{\omega h}{\sqrt{|\ell|}}\right)\right] & \text { if } \ell<0 .\end{cases}
$$

In the numerical outputs that follow we have used the data $Y=10, \nu=1 / 3$ for the Young modulus and the Poisson ratio of the material, respectively, and $h=1$. Figure 2 displays the work $W$ in function of $\ell$. Clearly, choosing $\ell>0$ is unphysical, since incompatible deformations produce less work that in the purely elastic case $(\ell \rightarrow \infty)$. In contrast, choosing $\ell<0$ is consistent with our expectation from the energetic point of view, the difference of work between the inelastic (irreversible) and elastic (reversible) transformations being the dissipation, see [2,4] for details.

Figure 3 shows the functions $\varphi$ and $\psi$ for some negative values of $\ell$. For $\ell \rightarrow-\infty$, the classical elastic solution is retrieved.

Figure 4 shows the same functions calculated with $\ell$ variable in space, namely $\ell=-1$ in the interval $[-0.1,0.1]$ and $\ell=-1000$ elsewhere. Although the domain is unbounded, the horizontal compression suggests that the model is able to predict necking phenomena. Let us stress once more that we have restricted ourselves to linearized equations. How to deal with finite deformations is briefly discussed thereafter. 

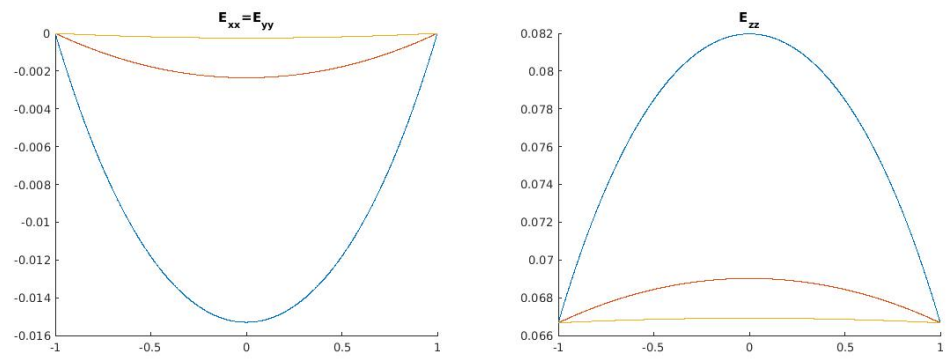

Figure 3. Planar (left) and vertical (right) deformations for $\ell=-10$ (blue), $\ell=-100$ (red), $\ell=-1000$ (yellow).
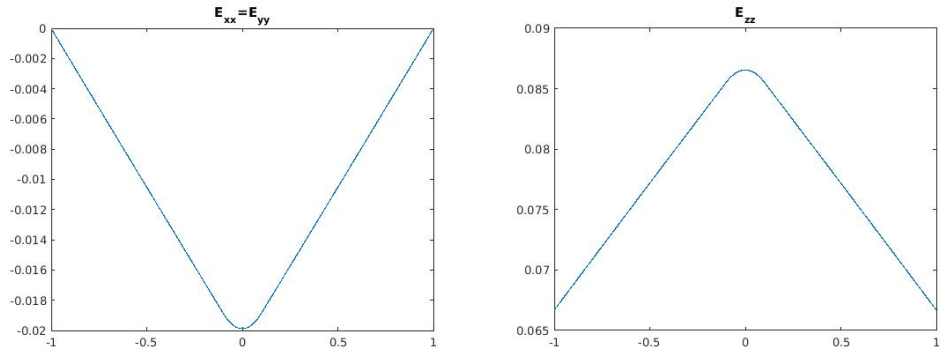

Figure 4. Planar (left) and vertical (right) deformations for $\ell$ variable in space.

9.7. Incremental formulation of hardening problems. An incremental formulation consists in introducing a continuous family of loads, here denoted by $\left(\mathbb{K}_{t}\right)$, parameterized by a fictitious time $t$, starting from $\mathbb{K}_{0}=0$ and reaching the target value $\mathbb{K}_{T}=\mathbb{K}$ at final time $T$. The interval $[0, T]$ is split into subintervals $\left[t_{k}, t_{k+1}\right]$, and within each subinterval one solves the tangent problem (9.10). Here $\mathbb{A}$ and $\ell$ are tangent moduli, and $E$ is the strain increment. Nonlinear phenomena occur when these moduli vary between two increments. In the first increment, for small load, the behavior is usually elastic: $|\ell|$ is taken very large, one can even solve the standard elasticity equations. At some point, according to some yield stress or energetic criterion, nonlinearity and irreversibility appear: $|\ell|$ should be locally decreased. As stipulated by the second principle of Thermodynamics, this modification of the incompatibility modulus must be associated with a dissipation of free energy. The procedure is repeated in the following increments. Of course, in case of elastic unloading, $\ell$ should be again taken everywhere "infinite".

The process of update of $\ell$ (and also $\mathbb{A}$ ) is obviously not completely determined. The point is to represent the complex hardening phenomenon. This will be investigated in future works. At least to comply with the Second Principle a sensitivity analysis of the free energy with respect to local perturbations of $\ell$ may be carried out. It has been done in [4] for the model (9.10) reduced to its principle part, i.e. (8.8). The full model is under scrutiny.

Acknowledgements. The second author was supported by national funding from FCT - Fundaçâo para a Ciência e a Tecnologia, under the project: UID/MAT/04561/2019 as well as by the FCT Starting Grant " Mathematical theory of dislocations: geometry, analysis, and modelling" (IF/00734/2013).

\section{REFERENCES}

[1] R. Abu Al-Rub. Continuum-based modeling of size effects in micro-and nanostructured materials. In Handbook of Micromechanics and Nanomechanics. Pan Standford Publ., 2013.

[2] S. Amstutz and N. Van Goethem. Existence results for an intrinsic model of incompatible elasticity. hal report: hal-02045046, 2019. 
[3] S. Amstutz and N. Van Goethem. Analysis of the incompatibility operator and application in intrinsic elasticity with dislocations. SIAM J. Math. Anal., 48(1):320-348, 2016.

[4] S. Amstutz and N. Van Goethem. Incompatibility-governed elasto-plasticity for continua with dislocations. Proc. R. Soc. A, 473(2199), 2017.

[5] G. Anzellotti and M. Giaquinta. On the existence of the fields of stresses and displacements for an elastoperfectly plastic body in static equilibrium. J. Math. Pures Appl., 61:219-244, 1982.

[6] G. Anzellotti and S. Luckhaus. Dynamical evolution of elasto-perfectly plastic bodies. Appl. Math. Optim., 15(6):121-140, 1987.

[7] M. Navier Barr de Saint-Venant, A. J. C. Premire section : De la rsistance des solides par navier. - 3e d. avec des notes et des appendices par $\mathrm{m}$. barr de saint-venant. tome 1. In Rsum des leons donnes lEcole des Ponts et Chausses sur lapplication de la mcanique ltablissement des constructions et des machines. Dunod, Paris, 1864.

[8] Reddy B.D, Ebobisse F., and McBride. A. Well-posedness of a model of strain gradient plasticity for plastically irrotational materials. Int. J. of Plast., 24(5), 2008.

[9] E. Beltrami. Sull'interpretazione meccanica delle formule di Maxwell. Mem. dell'Accad. di Bologna, 7:1-38, 1886.

[10] B. A. Bilby, R. Bullough, and E. Smith. Continuous distribution of dislocations: a new application of the methods of non-riemannian geometry. Proc. R. Soc. A, 231(1):263-273, 1955.

[11] J. F. Bonnans and A. Shapiro. Perturbation analysis of optimization problems. Springer Series in Operations Research. Springer-Verlag, New York, 2000.

[12] E. Cartan. Sur une generalisation de la notion de courbure de Riemann et les espaces a torsion. C. R. Acad. Sci. Paris, 174:593-597, 1922.

[13] E. Cesaro. Sulle formole del Volterra, fondamentali nella teoria delle distorsioni elastiche. Rend. Accad. R. Napoli, 12:311-321, 1906.

[14] P. G. Ciarlet. An introduction to differential geometry with applications to elasticity. J. Elasticity, 78-79(13):3-201, 2005.

[15] Ph. G. Ciarlet and C. Mardare. Intrinsic formulation of the displacement-traction problem in linearized elasticity. Math. Models Methods Appl. Sci., 24(6):1197-1216, 2014.

[16] F. Cosserat, E. Cosserat. Théorie des corps déformables. A. Hermann et Fils, Paris, 1909.

[17] G. Dal Maso, A. De Simone, and M. G. Mora. Quasistatic evolution problems for linearly elastic-perfectly plastic materials. Arch. Ration. Mech. Anal., 180(2):237-291, 2006.

[18] E. Davoli and G. A. Francfort. A critical revisiting of finite elasto-plasticity. SIAM J. Math. Anal., 47(1):526565,2015

[19] B. A. Dubrovin, A. T. Fomenko, and S. P. Novikov. Modern geometry - methods and applications, Part 1 (2nd edn). Cambridge studies in advanced mathematics. Springer-Verlag, New-York, 1992.

[20] G. Duvaut. Mécanique des milieux continus. Collection Mathématiques appliquées pour la maitrise. Masson, 1990.

[21] F. Ebobisse and P. Neff. Existence and uniqueness for rate-independent infinitesimal gradient plasticity with isotropic hardening and plastic spin. Math. Mech. Solids, 15(6):691-703, 2010.

[22] M. Epstein. The Geometrical Language of Continuum Mechanics. Cambridge University Press, 2010.

[23] L. Euler. Recherces sur la courbure des surfaces. Mem. Acad. Sci. Berlin, 16:119-143, 1767.

[24] P. Neff F. Ebobisse. Existence and uniqueness for rate-independent infinitesimal gradient plasticity with isotropic hardening and plastic spin. Mathematics and Mechanic of Solids, 14(8), 2009.

[25] F. Flaherty and M.P. do Carmo. Riemannian Geometry. Mathematics: Theory \& Applications. Birkhäuser Boston, 2013.

[26] N. A. Fleck and J. W. Hutchinson. A reformulation of strain gradient plasticity. J. Mech. Phys. Solids, 49(10):2245-2271, 2001.

[27] A. E. Green and W. Zerna. Theory of elasticity in general coordinates (finite strain). Phil. Mag., 41:313-336, 1950.

[28] H. J. Greenberg. Complementary minimum principles for an elastic -plastic material. Q. Appl. Math., 7:85-95, 1949.

[29] M. E. Gurtin. Configurational Forces as Basic Concepts of Continuum Physics. Applied Mathematical Sciences, 137. Springer, 2000.

[30] M. E. Gurtin and L. Anand. A theory of strain-gradient plasticity for isotropic, plastically irrotational materials. i: small deformations. J. Mech. Phys. Solids, 53(7):1624-1649, 2005.

[31] M. E. Gurtin and A. Needleman. Boundary conditions in small-deformation, single-crystal plasticity that account for the Burgers vector. J. Mech. Phys. Solids, 53(1):1-31, 2005.

[32] W. Han and B. D. Reddy. Plasticity. Mathematical theory and numerical analysis. 2nd ed. Springer, New-York, 2013.

[33] R. Hardt and D. Kinderlehrer. Elastic plastic deformation. Appl. Math. Optim., 10:213-246, 1983.

[34] R. Hill. Constitutive dual potentials in classical plasticity. Journal of the Mechanics and Physics of Solids, $35(1): 23-33,1987$. 
[35] D. Hull and D.J. Bacon. Introduction to Dislocations. Materials science and technology. Elsevier Science, 2011.

[36] C. Johnson. Existence theorems for plasticity problems. J. Math. Pures Appl., 55:431-444, 1976.

[37] G. Kirchhoff. Vorlesungen über Mechanik. Lecture notes in physics, 47. Birkhäuser, Basel, Leipzig, 1876.

[38] H. Kleinert. Gauge fields in condensed matter, Vol.1. World Scientific Publishing, Singapore, 1989.

[39] K. Kondo. Non-riemannian geometry of the imperfect crystal from a macroscopic viewpoint. in RAAG Memoirs of the unifying study of basic problems in engineering sciences by means of geometry, Vol.1, Division D, Gakuyusty Bunken Fukin-Day, pages 458-469, 1955.

[40] H. Kozono and T. Yanagisawa. $L^{r}$-variational inequality for vector fields and the Helmholtz-Weyl decomposition in bounded domains. Indiana Univ. Math. J., 58(4):1853-1920, 2009.

[41] E. Kröner. Continuum theory of defects. In R. Balian, editor, Physiques des défauts, Les Houches session XXXV (Course 3). North-Holland, Amsterdam, 1980

[42] J E. Kröner. $C^{1}$ isometric imbeddings. Int. J. Solids and Structures, 29(14/15):1849-1257, 1992.

[43] N. H. Kuiper. On $C^{1}$-isometric imbeddings. I, II. Nederl. Akad. Wet., Proc., Ser. A, 58:545-556, 683-689, 1955.

[44] M. Lazar and G. A. Maugin. Nonsingular stress and strain fields of dislocations and disclinations in first strain gradient elasticity. Int. J. Eng. Sci., 43:1157-1184, 2005

[45] A. E. H. Love. A treatise on the mathematical theory of elasticity. Cambridge University Press, 1892.

[46] G. Maggiani, R. Scala, and N. Van Goethem. A compatible-incompatible decomposition of symmetric tensors in $L^{p}$ with application to elasticity. Math. Meth. Appl. Sci, 38(18):5217-5230, 2015.

[47] G.A. Maugin. The method of virtual power in continuum mechanics: Application to coupled fields. Acta Mech. $35: 1-70,1980$

[48] J. H. Michell. On the direct determination of stress in an elastic solid, with application to the theory of plates. Proc. Lond. Math. Soc., s131:100124, 1906.

[49] A. Mielke and T. Roubíček. Rate-independent elastoplasticity at finite strains and its numerical approximation. Math. Models Methods Appl. Sci., 12:2203-2236, 2016.

[50] A. Mielke and U. Stefanelli. Linearized plasticity is the evolutionary $\gamma$-limit of finite plasticity. J. Eur. Math. Soc. (JEMS), 15(3):923-948, 2013.

[51] R. D. Mindlin. Micro-structure in linear elasticity. Arch. Ration. Mech. Anal., 16:51-78, 1964.

[52] J. J. Moreau. Application of convex analysis to the treatment of elastoplastic systems. In Applications of methods of functional analysis to problems in mechanicss. Springer, Berlin, 1976.

[53] G. Müller and J. Friedrich. Challenges in modeling of bulk crystal growth. J. Cryst. Growth, 266(1-3):1-19, 2004

[54] J. Nash. $C^{1}$ isometric imbeddings. Ann. Math. (2), 60:383-396, 1954.

[55] W. Noll. Materially uniform bodies with inhomogeneities. Arch. Rational Mech. Anal., 27:1-32, 1967.

[56] P. Nunes. Petri nonni salaciensis opera, basileae. Biblioteca Nacional de Portugal, Reediçãao e tradução, com comentários, pela Academia de Ciências de Lisboa Fundação Calouste Gulbenkian, Lisboa, 2008 e $2011,1566$.

[57] J. R. Rice. On the structure of stress-strain relations for time-dependent plastic deformation in metals. Trans ASME. J. Appl. Mech, page 728, 1970.

[58] B. Riemann. Über die hypothesen, welche der geometrie zu grunde liegen. in Riemann's Gesamm. Math. Werke XIII, Abhandl. Kgl. GeseU. Wiss. GSttingen XIII:272-287, 1868.

[59] R. Scala and N. Van Goethem. Currents and dislocations at the continuum scale. Methods Appl. Anal., 23(1):134, 2016.

[60] R. Scala and N. Van Goethem. Geometric and analytic properties of dislocation singularities. Proc. Roy. Soc. Edinb Sect. A, 149(4), 2019.

[61] R. Scala and N. Van Goethem. A variational approach to single crystals with dislocations. SIAM J. Math. Anal., 51(1):489-531, 2019.

[62] J. A. Schouten. Ricci-Calculus (2nd edn). Springer Verlag, Berlin, 1954.

[63] W. R. Seugling. Equations of compatibility for finite deformation of a continuous medium. The American Mathematical Monthly, 57(10):679-681, 1950

[64] J.C. Simo and T.J.R. Hughes. Computational inelasticity. Springer, Berlin, 1998.

[65] B. Sun. Incompatible deformation field and riemann curvature tensor. Applied Mathematics and Mechanics, 38(3):311-332, Mar 2017.

[66] P.-M. Suquet. Sur les équations de la plasticité: existence et régularité des solutions. J. Méc., Paris, 20:3-39, 1981.

[67] E.B. Tadmor, R.E. Miller, and R.S. Elliott. Continuum Mechanics and Thermodynamics: From Fundamental Concepts to Governing Equations. Cambridge University Press, 2011.

[68] R. Temam. Problèmes mathématiques en plasticité. J. Phys. Chem. Solids, 69:320-324, 2008.

[69] R. Thom. La vie et l'œuvre de Hassler Whitney. (Life and work of Hassler Whitney). C. R. Acad. Sci., Paris, Sér. Gén., Vie Sci., 7(6):473-476, 1990.

[70] N. Van Goethem. The non-Riemannian dislocated crystal: a tribute to Ekkehart Kröner's (1919-2000). J. Geom. Mech., 2(3), 2010. 
[71] N. Van Goethem. Strain incompatibility in single crystals: Kröner's formula revisited. J. Elast., 103(1):95-111, 2011.

[72] N. Van Goethem. The Frank tensor as a boundary condition in intrinsic linearized elasticity. J. Geom. Mech., 8(4):391-411, 2016.

[73] N. Van Goethem. Incompatibility-governed singularities in linear elasticity with dislocations. Math. Mech. Solids, 22(8):16881695, 2017.

[74] N. Van Goethem, A. de Potter, N. Van den Bogaert, and F. Dupret. Dynamic prediction of point defects in Czochralski silicon growth. An attempt to reconcile experimental defect diffusion coefficients with the $V / G$ criterion. J. Phys. Chem. Solids, 69:320-324, 2008.

[75] N. Van Goethem and F. Dupret. A distributional approach to $2 D$ Volterra dislocations at the continuum scale. Europ. Jnl. Appl. Math., 23(3):417-439, 2012.

[76] V. Volterra. Sulle equazioni differenziali lineari: nota. Rend. Mat. Acc. Lincei, 4(3), 1887.

[77] V. Volterra. Sur l'équilibre des corps élastiques multiplement connexes. Ann. Sci. École Norm. Sup., 3(24):401$517,1907$.

[78] V. Volterra. Sulle equazioni integro-differenziali della teoria dell'elasticitá. Rend. Mat. Acc. Lincei, 5(18):1295$301,1909$.

[79] C. C. Wang. On the geometric structure of simple bodies, a mathematical foundation for the theory of continuous distributions of dislocations. Arch. Rational Mech. Anal., 27(1):33-94, 1967.

[80] H. Weyl. Die Idee der Riemannschen Fläche. B. G. Teubner, Leipzig, 1 edition. 2 edn, B. G. Teubner, Leipzig, 1923; Reprint of 2 edn, Chelsea Co., New York, 1951; 3 edn, revised, B. G. Teubner, Leipzig, 1955. English translation of 3 edn, The Concept of a Riemann Surface, Addison-Wesley, 1964. Dover edition 2009., 1951.

[81] H. Whitney. Topological properties of differentiable manifolds. Bull. Am. Math. Soc., 43:785-805, 1937.

[82] A. Yavari and A. Goriely. Riemann-cartan geometry of nonlinear dislocation mechanics. Archive for Rational Mechanics and Analysis, 205(1):59-118, Jul 2012.

[83] A. Yavari and A. Goriely. Non-metricity and the nonlinear mechanics of distributed point defects. In G.-Q. G. Chen, M. Grinfeld, and R. J. Knops, editors, Differential Geometry and Continuum Mechanics, pages 235-251, Cham, 2015. Springer International Publishing.

Université D'Avignon, Laboratoire de Mathématiques D'Avignon, 301 rue Baruch de Spinoza, 84916 Avignon, France

E-mail address: samuel.amstutz@univ-avignon.fr

Universidade de Lisboa, Faculdade de Ciências, Departamento de Matemática, CMafcio, Alameda da Universidade, C6, 1749-016 Lisboa, Portugal

E-mail address: vangoeth@fc.ul.pt 